\title{
MATRIX REMODELING ACCOMPANIES IN VITRO ARTICULAR CARTILAGE SPHERICAL SHAPING
}

\author{
A Thesis \\ presented to \\ the Faculty of California Polytechnic State University, \\ San Luis Obispo
}

\author{
In Partial Fulfillment \\ of the Requirements for the Degree \\ Master of Science in Biomedical Engineering
}

By

Nathan Thomas Balcom

June 2013 
(C) 2013

Nathan Thomas Balcom

ALL RIGHTS RESERVED 
TITLE:

AUTHOR:

DATE SUBMITTED:

COMMITTEE CHAIR:

COMMITTEE MEMBER:

COMMITTEE MEMBER:
MATRIX REMODELING ACCOMPANIES IN VITRO ARTICULAR CARTILAGE SPHERICAL SHAPING

Nathan Thomas Balcom

June 2013
Dr. Scott Hazelwood, Associate Professor

Biomedical and General Engineering Department

Dr. Stephen Klisch, Professor

Mechanical and Biomedical Engineering Department

Dr. Lanny Griffin, Department Chair

Biomedical and General Engineering Department 


\title{
ABSTRACT \\ MATRIX REMODELING ACCOMPANIES IN VITRO ARTICULAR CARTILAGE SPHERICAL SHAPING
}

\author{
Nathan Thomas Balcom
}

\begin{abstract}
Introduction: Articular cartilage (AC) is a low friction load bearing material found in synovial joints. The natural repair of damaged tissue is difficult and often requires surgical intervention. With large defects it becomes necessary to match the original tissue geometry. We hypothesized that localized collagen (COL) and/or proteoglycan (PG) remodeling occurs during AC spherical reshaping. The objective of this study was to determine the presence, magnitude and depth dependence of COL and PG remodeling that accompanies AC reshaping. Methods: Full thickness AC blocks (7x7 $\mathrm{mm}^{2}$ surface area) were harvested from the ridges of the patellofemoral groove of immature (1-3 week old) bovine knees. The top 0-1 mm with intact articular surface was sliced off with a vibrating microtome. A $6 \mathrm{~mm}$ diameter disk was punched out of the slice and the most anterior edge was notched to mark directionality. The final sample was a 1 $\mathrm{mm}$ thick, $6 \mathrm{~mm}$ diameter disk with a notch on the most anterior edge. Samples were either not treated (day 0; D0) or allowed to free swell overnight in $20 \%$ FBS. Then cultured samples were placed in culture with $20 \%$ FBS in either free swelling (FS), static bending with the articular surface concave (concave) or in static bending with the articular surface convex (convex). Wet-weight and opening angle were measured before and 2 hours after removal from culture. Following culture, samples were cut in half in the anterior posterior direction. One half of each sample was frozen and later analyzed for PG, COL and cell content. The other half was fixed for 24-48 hours in $4 \%$ paraformaldehyde; samples were then transferred to $20 \%$ Hexabrix for 24 hours before imaging by micro-computed tomography $(\mu \mathrm{CT})$ to assess PG distribution. Following $\mu \mathrm{CT}$, samples were again placed in 4\% paraformaldehyde for 24-48 hours and then prepared for qPLM to assess collagen orientation $(\alpha)$, parallelism index (PI), and area fraction of non-birefringent tissue $\left(\mathrm{AF}_{\mathrm{NBR}}\right)$. Variations were assessed by ANOVA with post hoc tests for significant ANOVA $(\mathrm{p}<0.05)$. Results: Four days of spherical bending significantly changed $(\mathrm{p}<0.001)$ the outer opening angle of concave $\left(\gamma=144^{\circ}\right)$ and convex $\left(\gamma=152^{\circ}\right)$ samples when compared to both D0 $\left(\gamma=176^{\circ}\right)$ and FS $\left(\gamma=177^{\circ}\right)$ controls. There were no significant changes in PG, COL or cell content or concentration. Hexabrix concentration was significantly different in the D0 group between superficial and deep layers $(\mathrm{p}<0.01)$. Significant differences in area fraction were between the superficial and deep layers of D0 specimens $(\mathrm{p}<0.05)$ and between the superficial and deep layers of concave specimens ( $p<0.01)$. Discussion: Spherical bending reshapes AC into a cup shape. Trends of decreasing $\alpha$ standard deviation ( $\alpha$ SD) with depth in concave samples and increasing $\alpha \mathrm{SD}$ with depth in convex samples indicate that COL matrix disorganization is associated with regions of compressive strain. Consequently, further evaluation on the disorganization of the collagen network should be studied to elucidate mechanisms of cartilage reshaping.
\end{abstract}

Keywords: articular cartilage, spherical shaping, immature bovine, matrix remodeling 


\section{ACKNOWLEDGMENTS}

I would like to thank my advisors Dr. Stephen Klisch and Dr. Scott Hazelwood for their mentorship over the past three years. I feel honored to have worked with them and am thankful for the vast amount of effort they have dedicated to me and my projects.

Additionally, I would like to thank Dr. Robert Sah for his excellent mentorship in the lab and direction for the projects. Also thank you to Esther Cory, Barbara Schumacher, Van Wong, and Michelle Temple-Wong for answering the many questions I had daily while working in the lab, and special thanks to Elaine Chan and Dr. Chris Raub for allowing me to use their MATLAB codes for image analysis.

I would also like to thank my labmates, Dominick Grisafe and Juan FrancoGutierrez for keeping me somewhat normal and sharing in my frustrations while working long hours in the cartilage tissue engineering lab.

Finally, I would like to thank my lifelong friend Rod Marstell and my fiancée Amy Morales for reading over this thesis so many times and catching all the little errors. Thanks to my friends and family who have supported me so faithfully and who understood when I had to work for long hours.

Also thank you to Christ Jesus my God. To Him be the glory forever. Amen. This project was supported by NIH. 


\section{TABLE OF CONTENTS}

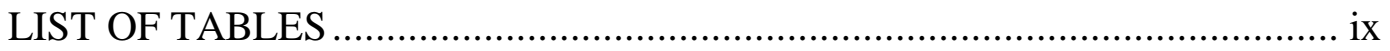

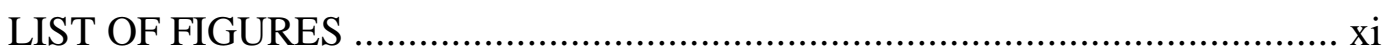

1. INTRODUCTION ....................................................................

1.1. Articular Cartilage Background ...................................................

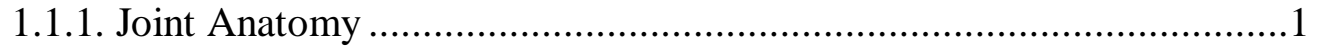

1.1.2. Composition of Articular Cartilage .................................................2

1.1.3. Microstructure ....................................................................

1.1.4. Variations in Microstructure with Immature Articular Cartilage ..........5

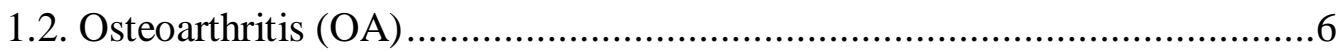

1.2.1. Epidemiology of OA ..............................................................6

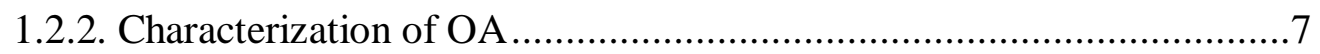

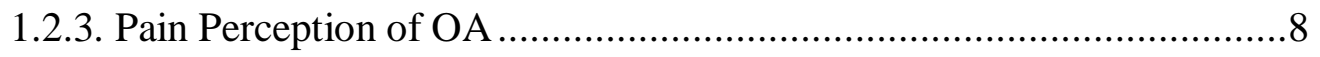

1.2.4. Current Repair Strategies ...................................................... 9

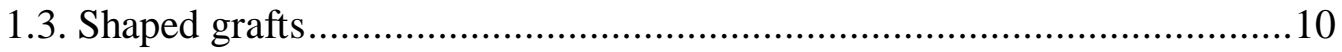

1.3.1. Necessity for shaped grafts .................................................. 10

1.3.2. Previous types of shaping ................................................... 12

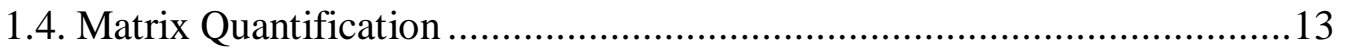

1.4.1. Micro-computed Tomography (Proteoglycans) .............................13

1.4.2. Quantitative Polarized Light Microscopy (Collagen) .......................14 
1.5. Study Objectives

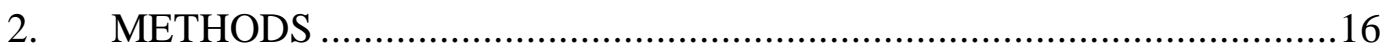

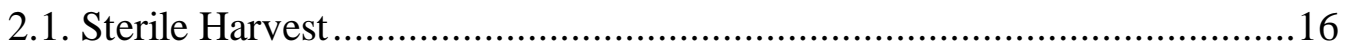

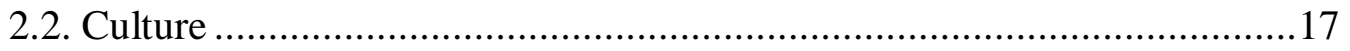

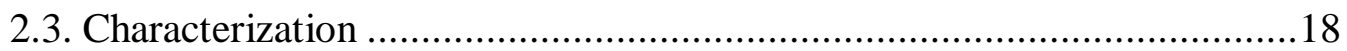

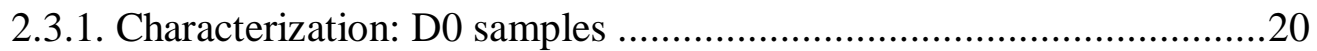

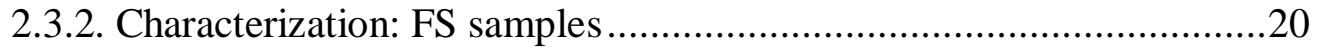

2.3.3. Characterization: concave and convex samples ................................20

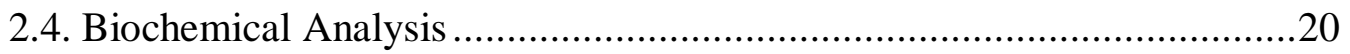

2.4.1. Biochemical Analysis: Proteinase K (ProK) digestion..........................20

2.4.2. Biochemical Analysis: Glycosaminoglycan (GAG) Assay ..................21

2.4.3. Biochemical Analysis: Hydroxyproline Assay ....................................21

2.4.4. Biochemical Analysis: DNA Assay ...............................................22

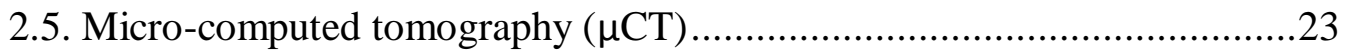

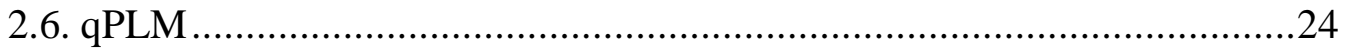

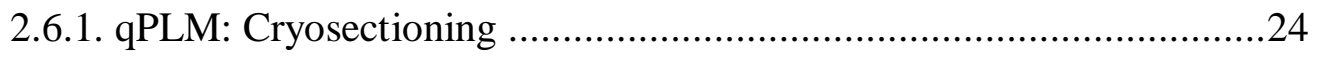

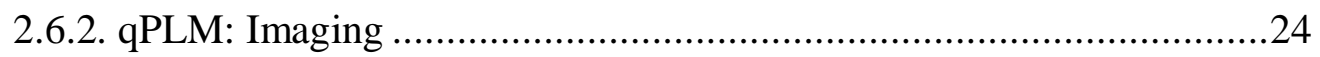

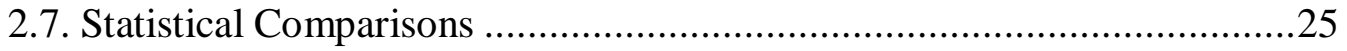

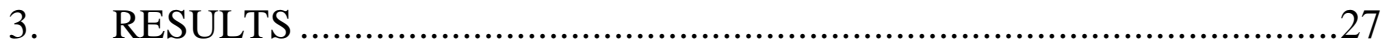

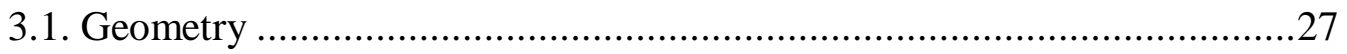


3.2. Biochemistry

3.3. Micro Computed Tomography ........................................................... 30

3.4. Quantitative Polarized Light Microscopy ......................................... 32

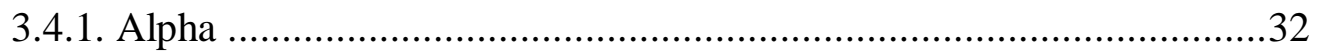

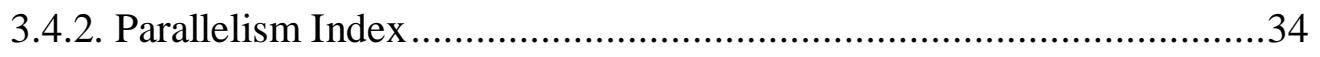

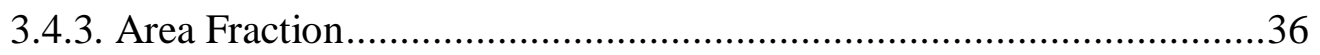

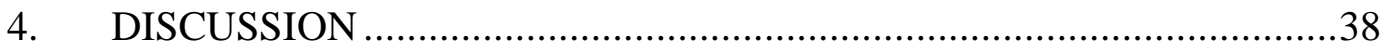

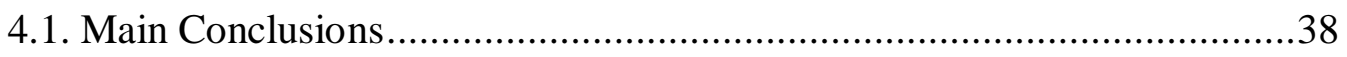

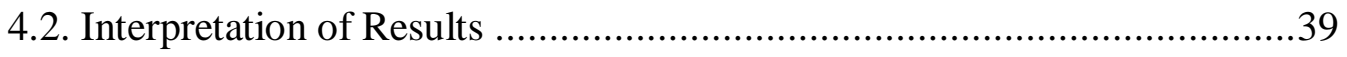

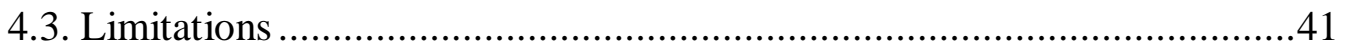

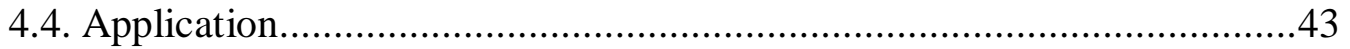

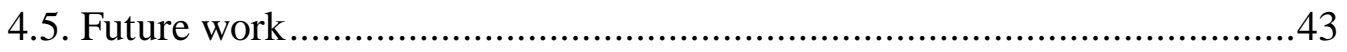

4.6. Conclusion .........................................................................44

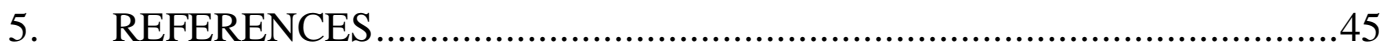

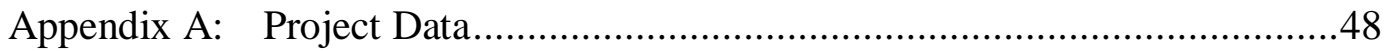




\section{LIST OF TABLES}

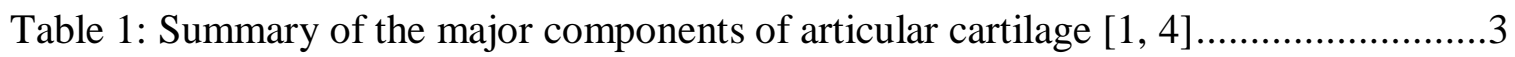

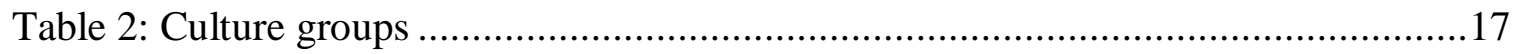

Table 3: p-values for 1-way ANOVA Hexabrix concentration (\%) comparing groups. Significant $\mathrm{p}$-values $(\mathrm{p}<0.05)$ are shown in BOLD.

Table 4: p-values for 1-way ANOVA Hexabrix concentration (\%) comparing layers within each group and using layers as a repeated measure. Significant $\mathrm{p}$-values $(\mathrm{p}<0.05)$ are shown in BOLD.

Table 5: p-values for 1-way ANOVA for collagen fiber orientation $(\alpha)$ and collagen fiber orientation standard deviation $(\alpha \mathrm{SD})$ comparing groups

Table 6: $\mathrm{p}$-values for 1-way ANOVA for $\alpha$ and $\alpha$ SD comparing layers within each group and using layers as a repeated measure. Significant p-values $(\mathrm{p}<0.05)$ are shown in BOLD.

Table 7: p-values for 1-way ANOVA for Parallelism Index (PI) and Parallelism Index Standard Deviation (PISD) comparing groups

Table 8: p-values for 1-way ANOVA for PI and PISD comparing layers within each group and using layers as a repeated measure. Significant p-values $(\mathrm{p}<0.05)$ are shown in BOLD.

Table 9: p-values for 1-way ANOVA for area fraction (AF) and area fraction standard deviation (AFSD) comparing groups

Table 10: p-values for 1-way ANOVA for AF and AFSD comparing layers within each group and using layers as a repeated measure. Significant p-values $(\mathrm{p}<0.05)$ are shown in BOLD.

Table 11: Relaxed opening angle data.

Table 12: Biochemical data normalized to either initial wet weight (WWi) or final wet weight (WWf)

Table 13: Hexabrix data for superficial (S), middle (M), and deep (D) layers. *Samples labeled by "Sample \#\#" indicate unknown sample name but known sample group due to a recording error.

Table 14: Orientation $(\alpha)$ data for superficial (S), middle (M), and deep (D) layers. Red indicates excluded data due to processing error.

Table 15: Orientation standard deviation $(\alpha \mathrm{SD})$ for superficial $(\mathrm{S})$, middle $(\mathrm{M})$ and deep (D) layers. Red indicates excluded data due to processing error.

Table 16: Parallelism Index data for superficial (S), middle (M), and deep (D) layers 
Table 17: Parallelism Index Standard Deviation (PISD) data for superficial (S),

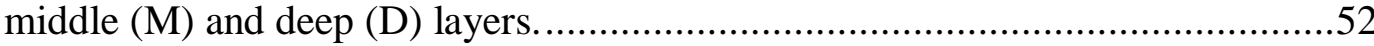

Table 18: Area fraction (AF) data for superficial (S), middle (M) and deep (D)

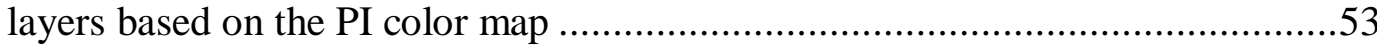

Table 19: Area fraction standard deviation (AFSD) data for superficial (S), middle (M) and deep (D) layers based on the PI color map. ..................................53 


\section{LIST OF FIGURES}

Figure 1: A. Typical synovial (diarthroidal joint) [3]. B. Knee joint (a diarthroidal joint) showing the applied loads and motion that the joint undergoes during normal use [1]. Note that ligaments and tendons are not explicitly shown in this figure. Figures from [3] and [1], respectively.

Figure 2: A. Keratan Sulfate and Chondrotin Sulfate chains covalently bound to a protein core also known as aggrecan. B. Multiple protein core (aggrecan) molecules bound to hyaluronan in a representation of a proteoglycan aggregate molecule. C. The interwoven structure of collagen and proteoglycans in articular cartilage [2].

Figure 3: A. Zonal architecture of the collagen network in mature articular cartilage with 3 distinct regions [2]. B. Zonal differences in cell phenotypes [6]. C. Proteoglycan content increases with cartilage tissue depth [7].

Figure 4: A. Depth-wise ellipse model representations of the maturation of collagen fibrils. Orientation is indicated by the major ellipse axes and PI is used to scale the aspect ratio. B. Variation in cellularity with depth in fetal, calf and adult cartilage. Figure from [5] and [8]

Figure 5: Prevalence of inactivity of Americans reported in 2002 where inactivity is defined as no reported leisure-time physical activity[9].

Figure 6: Current treatment paradigms for articular cartilage degeneration. Examples shown are for the femoral condyles of the knee [21].

Figure 7: Five categories of synovial joint geometries and examples of each [21]

Figure 8: Images of sterile harvest. A. Orange indicates the location of the patellofemoral groove on the knee. B. Bovine knee during harvest with example lines for cutting blocks. C. $6 \mathrm{~mm}$ diameter disk after slicing with a vibrating microtome; the most anterior corner has been cut to mark directionality.

Figure 9: A. Cross sectional loading of cartilage. B. Shape change quantified by inner opening angle $(\gamma)$ as defined previously[26]. C: Shape change quantified by outer opening angle $(\gamma)$. Figure adapted from [26].

Figure 10: Outer opening angle was calculated as an average of three measurements from pictures equally spaced around the cartilage disk. * Note that in this figure the opening angle is concave down, which is in contrast to Figure $9 \mathrm{C}$ that shows the outer opening angle as concave up. 
Figure 11: A. Representative 3D image of a Convex sample after imaged by micro CT. Purple represents the slice that was taken to create the image in B. The center 119 pixel width (highlighted in darker blue) represents C, which has been split into thirds for quantitative analysis.

Figure 12: A. Example of measurement to the surface normal. B. The axis used for each individual pixel is in reference to the direction of the line from that pixel to the nearest point on the surface of the tissue.

Figure 13: Representative images of samples in the relaxed state from each group. Note the distinct change in shape in Concave and Convex samples when compared to the unbent Day 0 and Free Swell samples. S, M and D indicate superficial, middle and deep layers, respectively.

Figure 14: A. Relaxed opening angle $\left(\gamma_{\text {relax }}\right)$ of Day 0 and cultured AC explants. B. Change in wet weight $(\Delta \mathrm{WW})$ of cultured explants. C. Water content of D0 and cultured explants. Symbols indicate $\mathrm{p}<0.05$ versus $*$ Day 0 and \#Free Swell. Data is shown as mean \pm SD. D0 $(n=9)$, Free Swell $(n=7)$, Concave $(n=3)$, and Convex $(n=7)$. Symbol inside concave and convex bars indicate which way the cartilage disk was bent with superficial side always up.

Figure 15: Glycosaminoglycan $(\mathrm{GAG})$ content $(\mathrm{A}, \mathrm{B})$; collagen $(\mathrm{COL})$ content $(\mathrm{C}$, $\mathrm{D})$; and cell content (E, F) of Day 0 and cultured explants normalized to initial wet weight (WWi; A, C, E), and final wet weight (WWf; B, D, F). For Day 0, WWi $=$ WWf. Data is shown as mean \pm SD. Day0 $(n=8)$, Free Swell $(n=6)$, Concave $(n=3)$, Convex $(n=6)$.

Figure 16: Hexabrix concentration for sample groups Day $0(n=4)$, Free Swell $(n=3)$, Concave $(n=2)$ and Convex $(n=5)$. Each group is split into 3 layers: superficial (S), middle (M) and deep (D). Data is shown as mean $\pm \mathrm{SD}$. * indicates significant differences between linked bars.

Figure 17: Orientation $\alpha$ (A) and standard deviation of orientation $\alpha \mathrm{SD}$ (B) for sample groups Day $0(n=2)$, Free Swell $(n=3)$, Concave $(n=3)$ and Convex $(n=3)$. Data is shown as mean \pm SD. * indicates significant differences between linked bars.

Figure 18: Parallelism index (A) and standard deviation of parallelism index (B) for sample groups Day 0, Free Swell, Concave and Convex $(n=3$ for all groups). Data is shown as mean $\pm \mathrm{SD}$. * indicates significant differences between linked bars.

Figure 19: Area Fraction (AF) (A) and Standard Deviation of Area Fraction (B) for sample groups Day 0, Free Swell, Concave, and Convex $(n=3$ for all groups). Data is shown as mean $\pm \mathrm{SD}$. * indicates significant differences ( $p$ <0.05) between linked bars using a post hoc Bonferroni test that accounts for repeated measures. 
Figure 20: A. Sample before bending B. Sample after bending. Dots represent proteoglycans which become closer together on the compression side and farther apart on the tensile side. Proteoglycans may not necessarily redistribute yet apparent changes in local concentration may appear....................40

Figure 21: Asymmetric strain distribution of articular cartilage in static uniaxial bending. Dashed line indicates midline of tissue sample. Dotted line indicates the neutral axis. Figure from [35]. 


\section{INTRODUCTION}

\subsection{Articular Cartilage Background}

Articular cartilage is a low friction, wear resistant, weight bearing material found on the ends of long bones $[1,2]$. It is a form of hyaline cartilage, which is characterized by a glassy smooth bluish-white appearance [3]. There are two other types of cartilage: elastic cartilage and fibrous cartilage. Elastic cartilage is found in the external ear and epiglottis, while fibrous cartilage is found in intervertebral disks and the menisci [3]. The rest of this background section will be focused on hyaline articular cartilage.

\subsubsection{Joint Anatomy}

The most common type of movable joint is the diarthroidal joint, which is characterized by the presence of a layer of articular cartilage that lines the opposing bony surfaces as well as a lubricating synovial fluid within a synovial cavity [2]. Figure 1A shows the typical anatomy of a diarthroidal joint. One example of this joint is the human knee. The knee has three separate surfaces to transfer loads: 1) medial femoral condyle to tibial plateau; 2) lateral femoral condyle to tibial plateau and 3) patella to the patellofemoral groove. (Articular cartilage in this study is harvested from the patellofemoral groove.) In each case the articular cartilage provides a nearly frictionless surface to transfer loads between surfaces. For both the medial and femoral condyles fibrocartilage disks, called menisci, rest in the joint to help maintain proper joint motion and provide stability along with the ligaments and tendons supporting the joint. Lubricating synovial fluid helps to reduce friction in the joint and is contained within a fibrous capsule that encloses the joint (Figure 1B) [1]. 


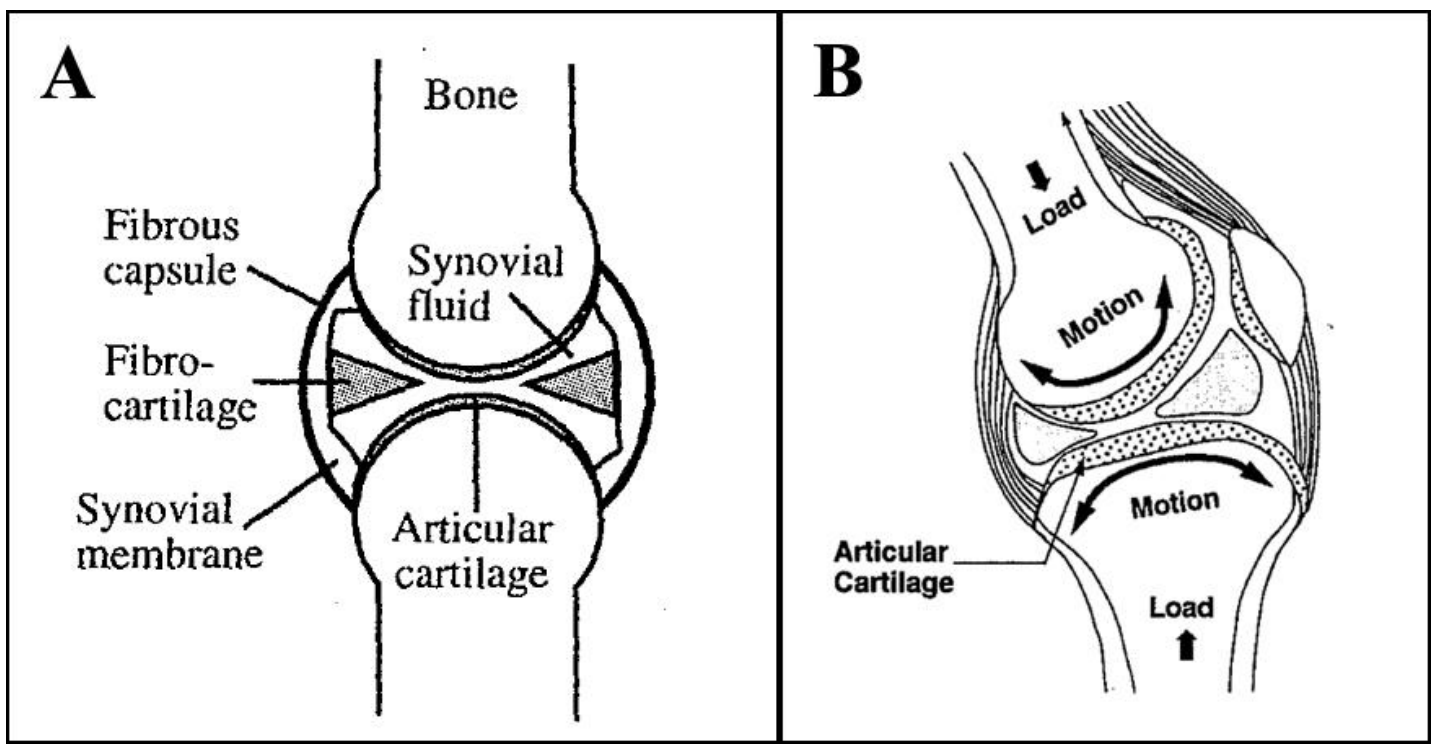

Figure 1: A. Typical synovial (diarthroidal joint) [3]. B. Knee joint (a diarthroidal joint) showing the applied loads and motion that the joint undergoes during normal use [1]. Note that ligaments and tendons are not explicitly shown in this figure. Figures from [3] and [1], respectively.

\subsubsection{Composition of Articular Cartilage}

From a biomechanical perspective, articular cartilage is a triphasic material ${ }^{\mathrm{a}}$ consisting of water, ions and a porous matrix [2]. Water fills the space between matrix molecules and makes up $68-85 \%$ of articular cartilage (Table 1). Positive ions in the water balance out the negatively charged proteoglycans (part of the porous matrix) and contribute slightly to the swelling of articular cartilage [2]. The structure and mechanical properties of articular cartilage are determined by the porous matrix. Interspersed throughout the matrix are chondrocytes (cartilage cells), which excrete the primary constituents of the matrix: type II collagen and proteoglycans. These two molecules are intertwined in articular cartilage (Figure 2C). Collagen exists as long fibers that support tensile loads [2]. Conversely, proteoglycans are large, negatively charged branched structures (Figure 2A \& B) that primarily support compressive loads [2]. Furthermore,

${ }^{a}$ While in reality cartilage can be thought of a triphasic material, for many modeling applications it is simply modeled as a biphasic material—a fluid filled porous matrix. 
the negative charges on the proteoglycans cause tissue swelling as the molecules repel each other. Expansion of the proteoglycans is limited by the tensile properties of the collagen network. Thus, the proteoglycans and collagen network complement each other to provide structure to articular cartilage.

Table 1: Summary of the major components of articular cartilage [1, 4]

\begin{tabular}{|l|c|}
\hline & Articular Cartilage \\
\hline Water (\% wet weight) & $68-85$ \\
\hline Collagen (\% wet weight; type II) & $10-20$ \\
\hline Proteoglycan (\% wet weight) & $5-10$ \\
\hline Cell Density (cells $\mathbf{~ 1 0}{\mathbf{~} \mathbf{m m}^{\mathbf{3}} \text { ) }}^{\mathbf{3}}$ & $14-334$ \\
\hline
\end{tabular}

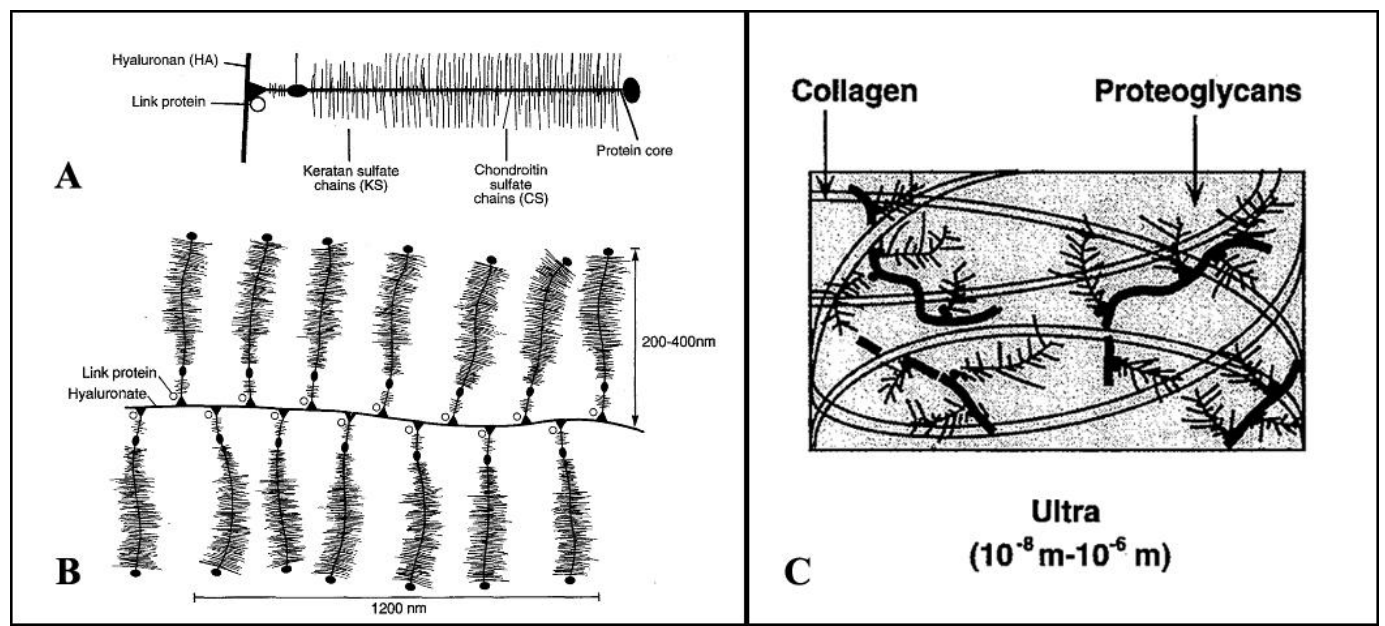

Figure 2: A. Keratan Sulfate and Chondrotin Sulfate chains covalently bound to a protein core also known as aggrecan. B. Multiple protein core (aggrecan) molecules bound to hyaluronan in a representation of a proteoglycan aggregate molecule. $C$. The interwoven structure of collagen and proteoglycans in articular cartilage [2].

\subsubsection{Microstructure}

The cartilage on the surface of a typical adult human joint is $2-4 \mathrm{~mm}$ thick and can be classified into 4 zones: superficial tangential zone, middle zone, deep zone and calcified zone (Figure 3; [2]). First, the superficial tangential zone is characterized by collagen fibers that run parallel to the articular surface. Additionally, the proteoglycan content is lowest in the superficial zone but increases in subsequent zones further from 
the surface. Second, in the middle zone the collagen fibers are randomly oriented and can be approximated as isotropic. This region also serves as a transition between the collagen fibers in the superficial tangential zone, which are horizontally oriented, to the collagen fibers in the deep zone, which are oriented perpendicular to the articular surface (Figure 3A). Finally, under the deep zone is the zone of calcified cartilage which transitions between the deep zone and subchondral bone. The distinction between deep zone and calcified cartilage can be viewed as the tide mark on histological sections. An important note is that immature bovine tissue is not completely developed [5]. This means the observed microstructure is different than adult tissue and inferences must be made accordingly. 


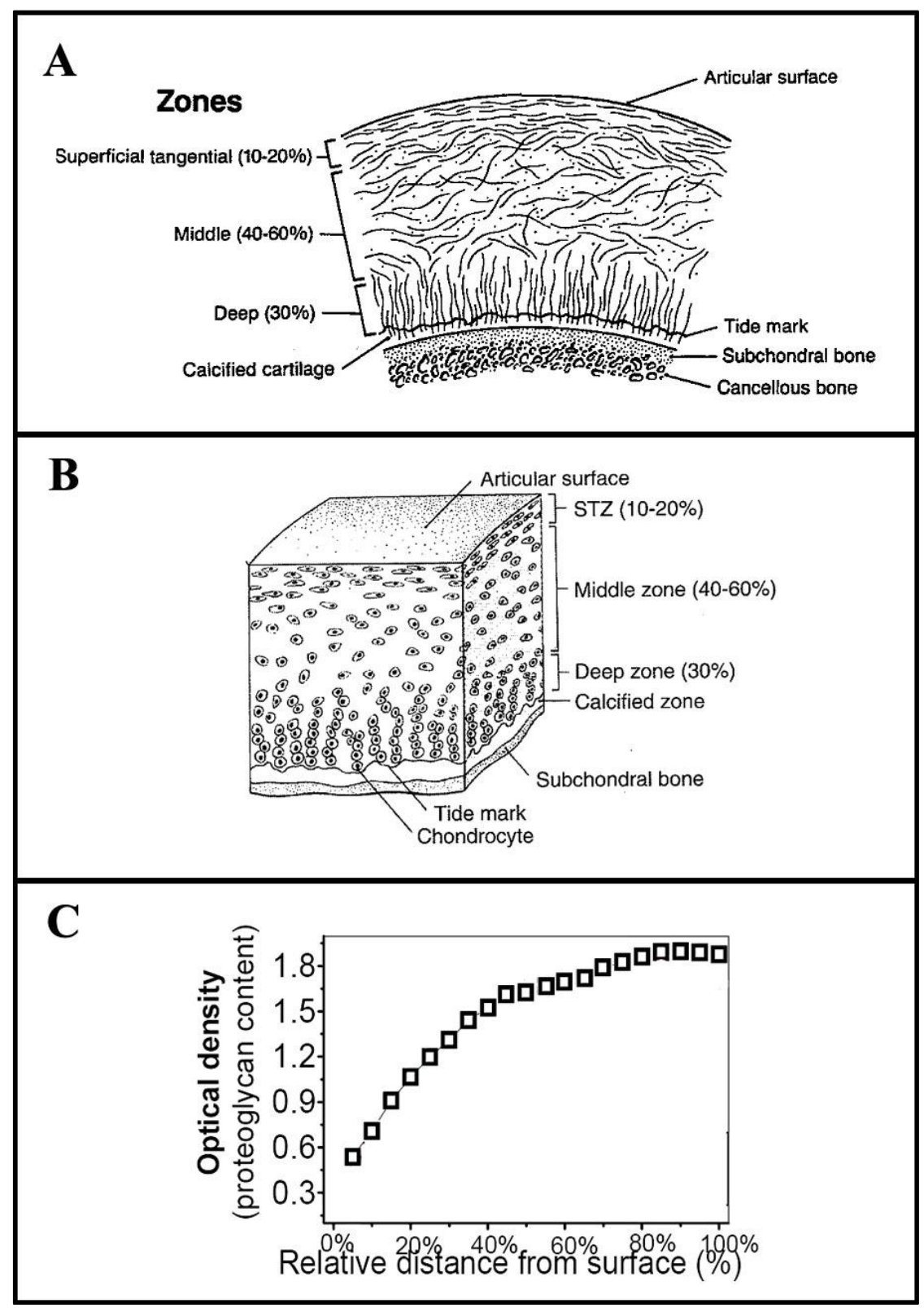

Figure 3: A. Zonal architecture of the collagen network in mature articular cartilage with 3 distinct regions [2]. B. Zonal differences in cell phenotypes [6]. C. Proteoglycan content increases with cartilage tissue depth [7].

\subsubsection{Variations in Microstructure with Immature Articular Cartilage}

Because this study involves immature tissue it is important to note some of the microstructural differences in immature tissue compared to adult cartilage. First of all the collagen network in immature tissue does not exhibit the four zones that adult cartilage can be classified into. Instead, most of the collagen fibers are horizontally oriented 
throughout the tissue depth (Figure 4A, [5]). There are also more total cells in younger tissue throughout the depth (Figure 4B). Nevertheless, the trend of higher cell content in more superficial tissue is consistent through all ages [8].

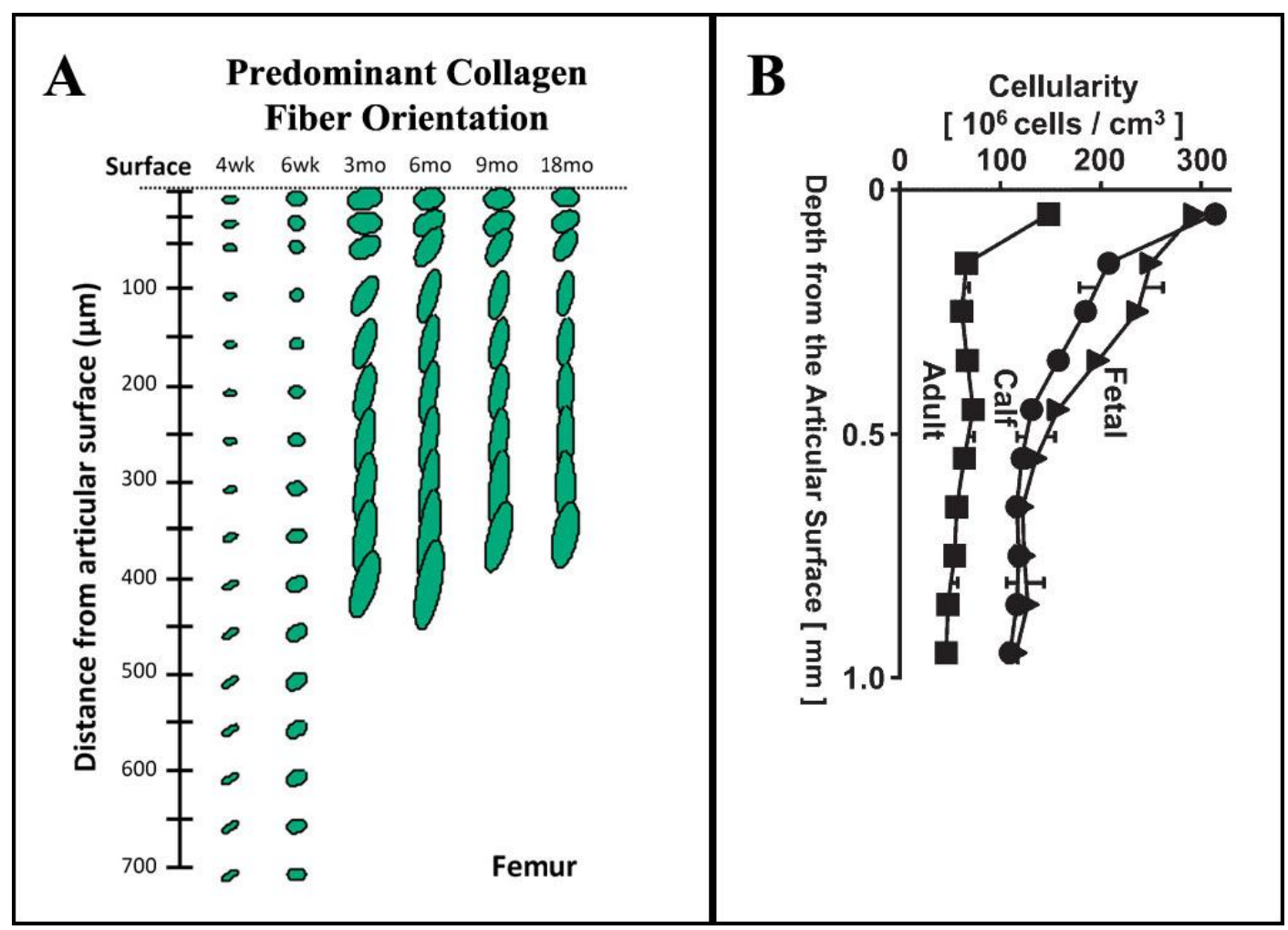

Figure 4: A. Depth-wise ellipse model representations of the maturation of collagen fibrils. Orientation is indicated by the major ellipse axes and PI is used to scale the aspect ratio. B. Variation in cellularity with depth in fetal, calf and adult cartilage.

Figure from [5] and [8]

\subsection{Osteoarthritis (OA)}

\subsubsection{Epidemiology of $\mathrm{OA}$}

Arthritis is the most common cause of disability in the United States affecting over $24 \%$ of women and over $18 \%$ of men. This means that an estimated 50 million Americans live with some form of diagnosed arthritis [9]. Additionally, it was reported that $50 \%$ of adults over the age of 65 had some form of arthritis between 2007 and 2009 [10]. While not all forms have the same symptoms, the common ones include pain and 
swelling around the joints [9]. The pain can be debilitating; in 2002, it was reported that the prevalence of physical inactivity was significantly higher in adults with arthritis [9] (Figure 5). The most relevant and common type is osteoarthritis.

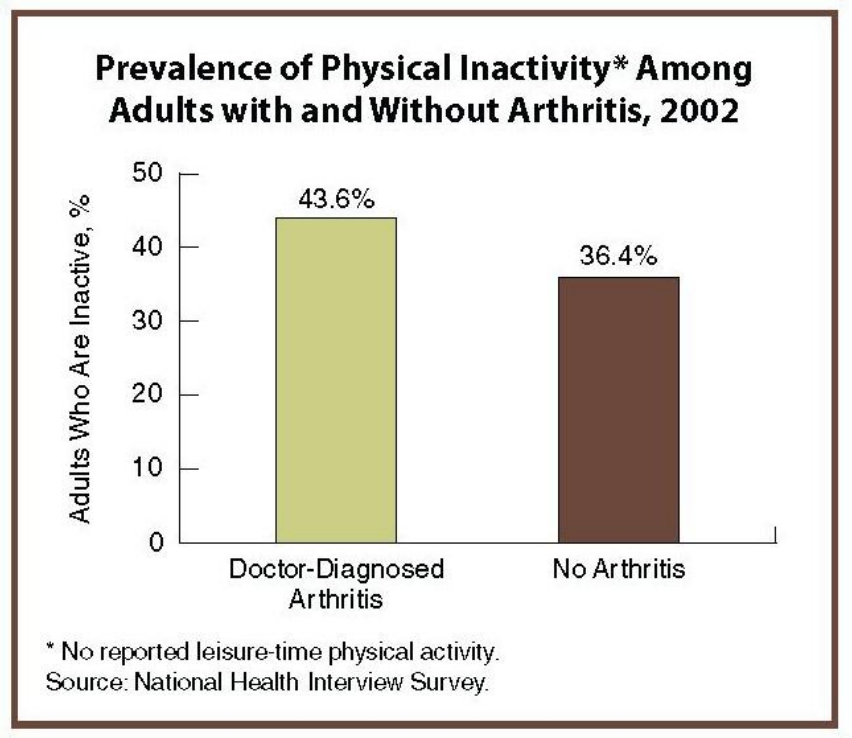

Figure 5: Prevalence of inactivity of Americans reported in 2002 where inactivity is defined as no reported leisure-time physical activity[9].

Osteoarthritis accounts for 55\% of all arthritis-related hospitalizations [11]. It is often associated with the wear and tear on the joints. Damage accumulates from excessive or recurrent joint loads from either working conditions or obesity. As the cartilage wears away, the joint swells and stiffens from inflammatory mechanisms. Consequently, the inflammatory mechanisms cause pain around the joint, and patient movement is limited because of pain or fear of pain $[9,11]$.

\subsubsection{Characterization of $\mathrm{OA}$}

Osteoarthritis has been characterized by cartilage degradation and other changes including subchondral bone cysts, subchondral sclerosis and osteophytes [12]. Early stages may also be characterized by increased protein synthesis and biosynthesis of matrix constituents as chondrocytes attempt to repair the damaged tissue that has been 
degraded away [12]. On a micro-scale, the cartilage in osteoarthritic joints is characterized by the depletion of proteoglycans from the cartilage extracellular matrix, and consequently, the mechanical properties of the tissue are altered [13]. Moreover, degradation of the collagen network in osteoarthritic cartilage means there is less restriction on the swelling pressure of the proteoglycans, and consequently, the cartilage swells [14]. It is also interesting to note that, while friction between joint surfaces exacerbates osteoarthritis, the early stage natural progression of osteoarthritis does not necessarily lead to an increase in friction [15]. If lubrication mechanisms such as interstitial fluid pressurization and boundary lubrication are still active, then the coefficient of friction does not increase with progressive osteoarthritis [15]. Nevertheless, there is an increase in friction in advanced stages of osteoarthritis especially when there is bone on bone contact [16].

\subsubsection{Pain Perception of OA}

A closer look at osteoarthritis reveals that the pain is not necessarily directly related to the amount of cartilage damage, but more so related to the surrounding joint tissues affected by the damage. Cartilage is an avascular, aneural tissue; therefore, the damaged cartilage cannot directly cause pain [12]. Instead, mechanisms for pain perception include the release of pro-inflammatory mediators (prostaglandins and cytokines), changes in the subchondral bone that may impinge on joint structures (osteophyte formation, bone sclerosis, subchondral cysts), and other joint pathologies (bone marrow lesions, effusions, and synovitis) [12]. These are often associated with other pathologies, but when the pain arises due to osteoarthritis it becomes important to repair or replace the damaged cartilage. If left untreated, not only can the patient become 
hypersensitive to the pain, but also continual wear can degrade the cartilage down to the subchondral bone [12]. Bone on bone contact in the osteoarthritic joint can elicit pain with very little movement or even at rest [16].

\subsubsection{Current Repair Strategies}

Focal defects of articular cartilage are treated differently depending on size and severity. Focal defects less than $2 \mathrm{~cm}^{2}$ are often treated by microfracture. Microfracture involves fracturing subchondral bone to stimulate mesenchymal stem cells to form a fibrocartilage repair tissue in the defect area [17]. For slightly larger defects $\left(2-3 \mathrm{~cm}^{2}\right)$, osteochondral autograft transplantation or autologous chondrocyte implantation (ACI) are commonly used $[18,19]$. Still larger defects $\left(\sim 4 \mathrm{~cm}^{2}\right)$, are commonly treated with allograft tissue [20]. Complications with allograft tissue may include the availability of donors, matching the geometry of the damaged sight and other immunologic responses [20]. As a last resort, joints with further degeneration require joint resurfacing or a total joint replacement (TJR) [21]. A summary of treatment options is displayed in Figure 6. 


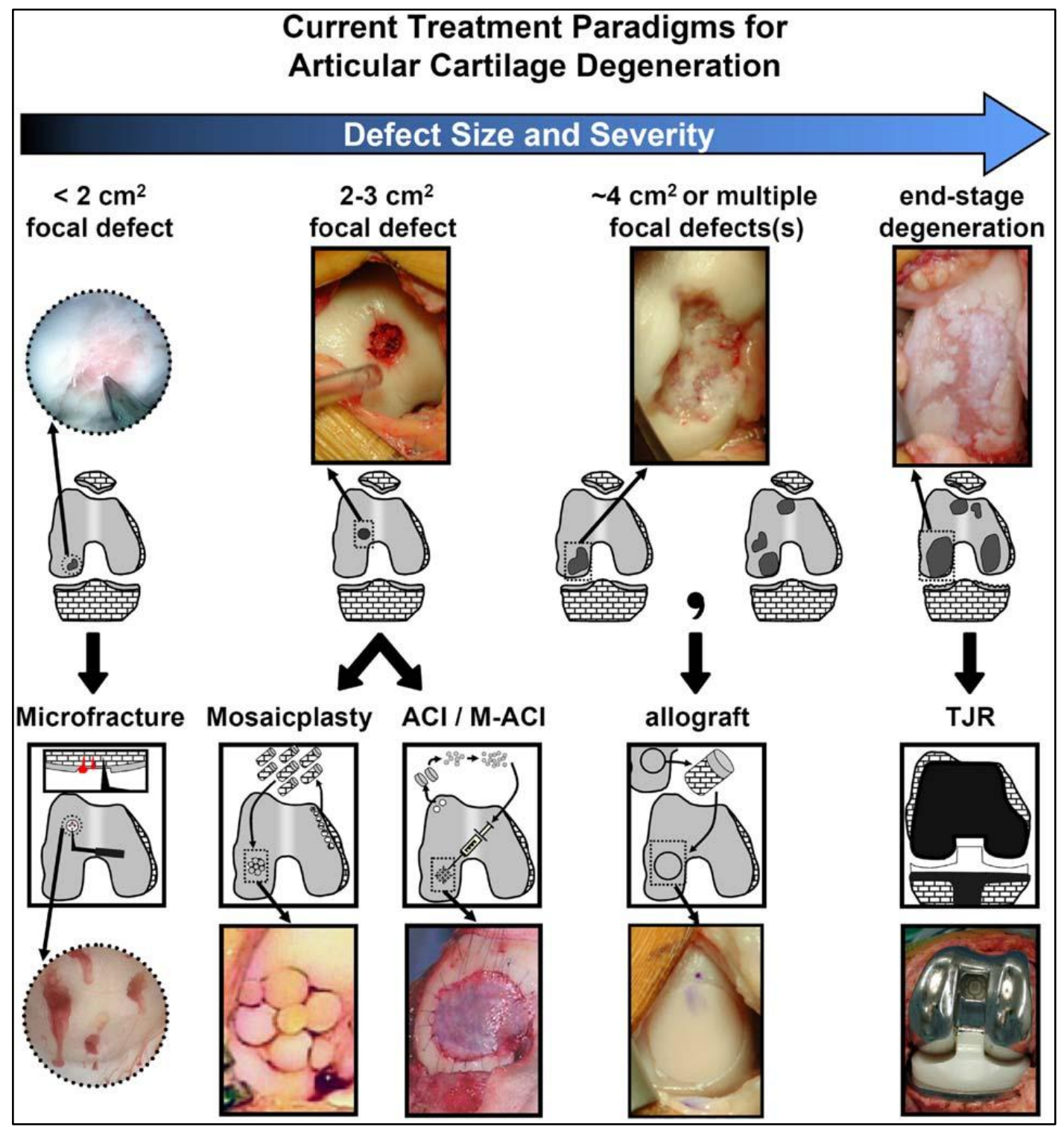

Figure 6: Current treatment paradigms for articular cartilage degeneration. Examples shown are for the femoral condyles of the knee [21].

\subsection{Shaped grafts}

\subsubsection{Necessity for shaped grafts}

The goal of tissue engineering is to replace damaged and dysfunctional tissue with tissue that matches the structure and function of healthy tissue. Synovial joints have various surface geometries that can be categorized into one of five categories: planar, cylindrical, spherical, elliptical, and saddle joints [21]. Examples of each are listed in 
Figure 7. The constant compression and sliding motion experienced in the joint means that the biomechanics of the joint must be maintained. Studies have shown that diseases that alter joint geometries produce increased wear and early osteoarthritis in the hip [22, 23]. With this in mind it is important to maintain native tissue geometry, especially knowing that altering the biomechanics of the joint could produce more harm than good. With careful matching of donor tissue, it is possible to replicate native tissue geometry without the need for shaped grafts [20]. Nevertheless, shaped articular cartilage grafts could allow damaged tissue to be replaced with autologous tissue without the complication of rejection found with allograft tissue. 


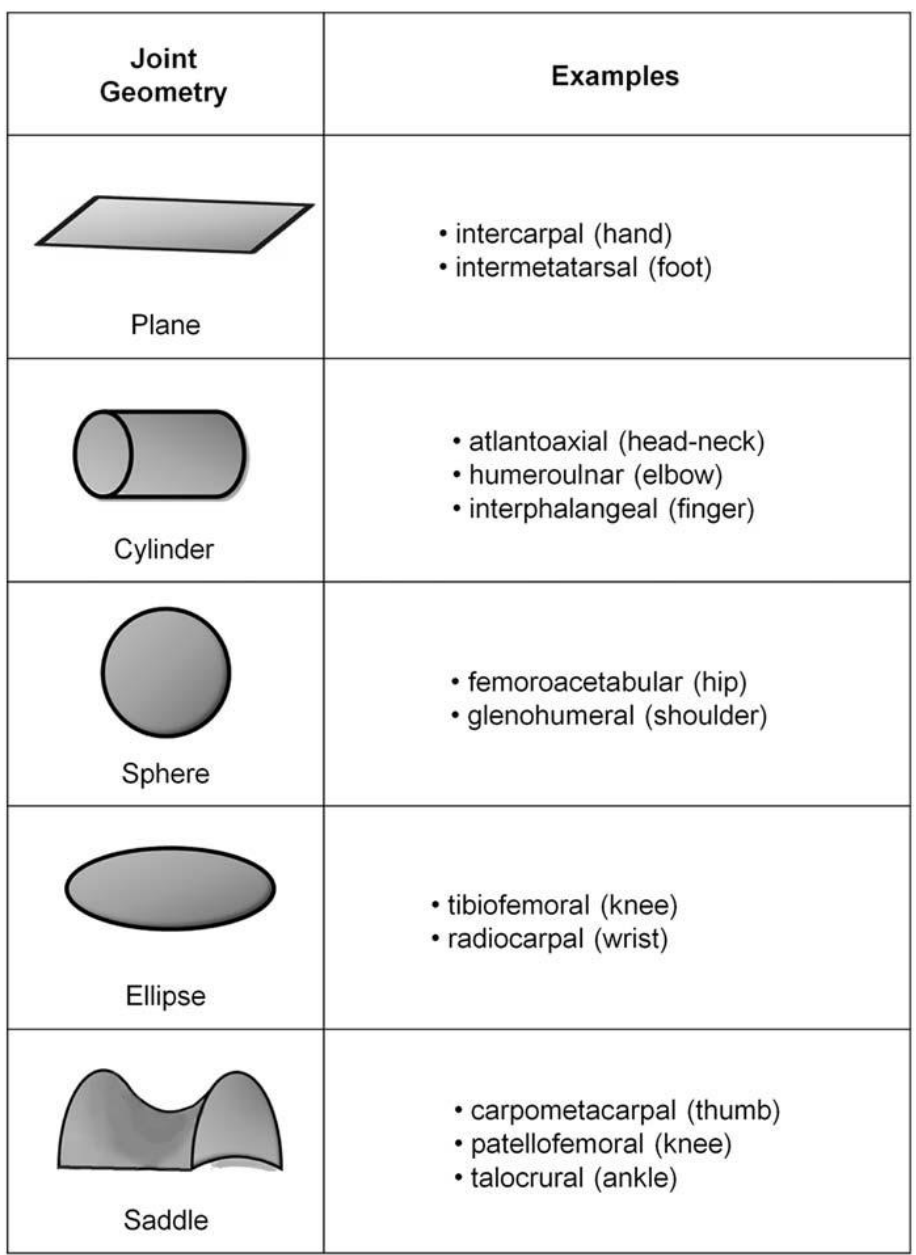

Figure 7: Five categories of synovial joint geometries and examples of each [21]

\subsubsection{Previous types of shaping}

Previous methods of shaping cartilage tissue have included thermoforming and electroforming. These methods cause the tissue to quickly reshape; however, chondrocyte viability is greatly reduced in the tissue [24]. Additionally, studies have shown that there is evidence of other electrochemical reactions which may affect the tissue in unknown ways [25].

Cartilage reshaping with in vitro mechanical loading is an alternative to these harmful methods. It has been shown that uniaxial 3-point bending using a cylindrical indenter reshaped articular cartilage after only 2 days of in vitro mechanical loading. 
Furthermore, the shape retention was significantly greater after 4 days in culture [26]. The study also showed that chondrocyte viability was maintained (>93\%) with static loading, and that the only damaged tissue was near the edges where the tissue explants had been cut. The study also showed that chondrocyte biosynthesis played a minimal role in cartilage reshaping, and suggested that metabolic reactions in the cartilage matrix play an important role in the reshaping of cartilage [26]. A recent study suggests that alterations in the collagen network are associated with cartilage reshaping. Regions of compression caused by static bending are associated with areas of matrix disorganization, while tensile strains on the convex side maintain matrix anisotropy in the collagen network [27]. This led to the conclusion that matrix disorganization in the compressive regions of uniaxial bent specimens contributes to the shape change [27].

\subsection{Matrix Quantification}

Using assays, it is possible to obtain average amounts of matrix constituents (proteoglycans and collagen); however, analyzing the distribution of these constituents requires different methods. In this study the distribution of proteoglycans is measured using contrast enhanced micro-computed tomography $(\mu \mathrm{CT})$. The collagen area fraction, orientation and parallelism index are measured using quantitative polarized light microscopy (qPLM).

\subsubsection{Micro-computed Tomography (Proteoglycans)}

Micro-computed tomography $(\mu \mathrm{CT})$ is an $\mathrm{x}$-ray based imaging technique that creates a high-resolution three-dimensional image. In most instances cartilage is not well defined using x-rays. To assess the distribution of proteoglycans in the cartilage explants it is common to use contrast enhanced $\mu \mathrm{CT}$. This method is based on the fact that 
proteoglycans are negatively charged and will repel a negatively charged contrast agent. In this case the contrast agent used is Hexabrix, a medical grade contrast agent commonly used in CT scans. By bathing the cartilage samples in Hexabrix and allowing the fluid inside the tissue to come to equilibrium, the Hexabrix distributes in a non-uniform fashion based on the repulsive forces of the proteoglycan network. When imaging the specimen, areas with a higher concentration of Hexabrix shows up as bright spots (high attenuation) [28]. The distribution of Hexabrix is inversely proportional to the distribution of proteoglycans. Consequently, by imaging the distribution of Hexabrix, it is possible to make inferences about the proteoglycan distribution.

\subsubsection{Quantitative Polarized Light Microscopy (Collagen)}

Distribution is the major factor that is being measured for proteoglycans. While the distribution is important for collagen, it is also important to map the orientation, parallelism, and area fraction of the collagen fibers. In order to map these parameters, quantitative polarized light microscopy (qPLM) is used.

Orientation $(\alpha)$ indicates the primary direction of collagen fibers with respect to the surface normal on a $-90^{\circ}$ to $90^{\circ}$ scale where $0^{\circ}$ is horizontal and parallel with the tissue top surface. Parallelism index (PI) indicates collagen fiber alignment in each pixel of the image. Because collagen fibers are much smaller than the resolution of the microscope, each pixel represents a number of collagen fibers. Parallelism index is on a scale of 0 to 1 , where 0 indicates the fibers are completely randomly oriented and 1 indicates all the fibers are aligned in the same direction. Area fraction of non-birefringent tissue $\left(\mathrm{AF}_{\mathrm{NBR}}\right)$ represents tissue that is more disorganized and/or has lower content of collagen (the major source of birefringence in articular cartilage). 


\subsection{Study Objectives}

There were four main objectives to this study. 1) To reshape a piece of articular cartilage into a "cup" shape, similar to the cup shape created previously, by culturing articular cartilage in spherical bending induced by a spherical indenter [29]. 2) To quantify and measure the geometry change, both during and following the application of the shape stimulus. 3) To determine the average contents of the matrix (proteoglycans, collagen and DNA) and water before and after tissue reshaping. 4) To characterize the microstructural remodeling of proteoglycans and collagen fibers using $\mu C T$ and qPLM, respectively. This study tests the hypothesis that in vitro shaping of immature cartilage explants via spherical static bending is accompanied by redistribution of the proteoglycan content and reorientation of the collagen network. 


\section{METHODS}

\subsection{Sterile Harvest}

Articular cartilage blocks, including subchondral bone, were harvested from the ridges of the patellofemoral groove of immature (1-3 week old) bovine knees (Figure 8A). The blocks had an approximate surface area of $7 \times 7 \mathrm{~mm}^{2}$. A $6 \mathrm{~mm}$ diameter core was punched through the top $\sim 2 \mathrm{~mm}$ of cartilage tissue. This ensured that a cylindrical cut was made through the superficial and middle layer of tissue, but not through the entire block. The block was then placed in the clamp of a vibrating microtome (Vibratome, St. Louis, MO), and the anterior edge was notched to mark the directionality of the $6 \mathrm{~mm}$ diameter core. (The notch on the final sample can be seen in Figure 8C). The block and core together were sliced to extract the superficial and middle layer $(0-1 \mathrm{~mm}$ depth from articular surface). This cut freed the $6 \mathrm{~mm}$ diameter core, so that the final sample was a 1 $\mathrm{mm}$ thick, $6 \mathrm{~mm}$ diameter disk (Figure 8C). Day 0 (D0) samples were characterized as described below and then cut in half in the anterior-posterior direction. One half of each sample was frozen at $-78^{\circ} \mathrm{C}$ until needed for biochemical analysis; the other half was fixed in $4 \%$ paraformaldehyde and stored at $4{ }^{\circ} \mathrm{C}$ in preparation for $\mu \mathrm{CT}$ and qPLM. 


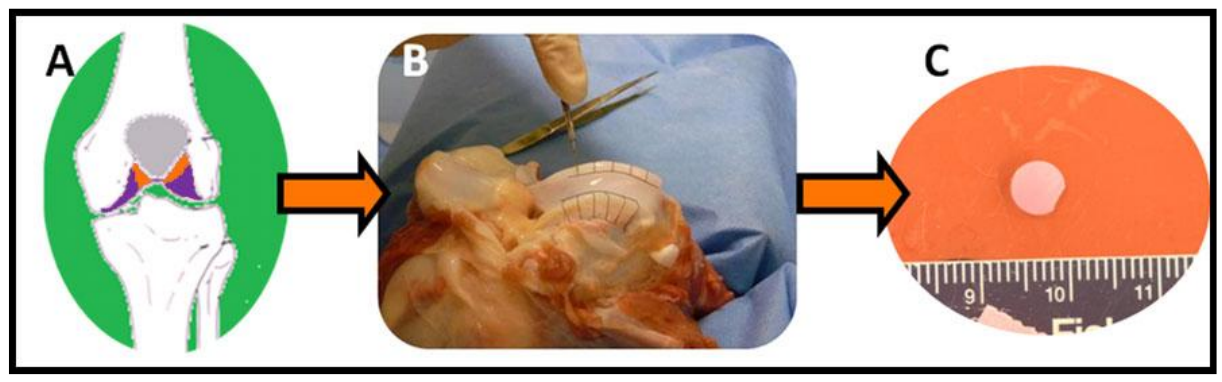

Figure 8: Images of sterile harvest. A. Orange indicates the location of the patellofemoral groove on the knee. B. Bovine knee during harvest with example lines for cutting blocks. C. 6 mm diameter disk after slicing with a vibrating microtome; the most anterior corner has been cut to mark directionality.

\subsection{Culture}

After harvest (day zero), cultured samples $\left(\mathrm{FS}^{\mathrm{b}}\right.$, concave, and convex) were allowed to free swell in a well plate overnight in $4 \mathrm{~mL}$ of $20 \% \mathrm{FBS}^{\mathrm{c}}$ at $37^{\circ} \mathrm{C}$. Then (day one) cultured samples were differentiated into their respective groups (Table 2). FS samples were placed in a new well plate. Concave samples were loaded in static spherical bending with the superficial layer concave. Convex samples were loaded in static spherical bending with the superficial layer convex. All cultured samples were immersed in $4 \mathrm{~mL}$ of $20 \% \mathrm{FBS}$ at $37^{\circ} \mathrm{C}$. The $20 \% \mathrm{FBS}$ was changed every other day during the 4 days of culture. The culture was terminated on day 5 .

Table 2: Culture groups

\begin{tabular}{|l|c|c|c|c|c|}
\hline Group & $\begin{array}{c}\text { Overnight } \\
\text { Free Swell }\end{array}$ & $\begin{array}{c}\text { Culture } \\
\text { Day 1-5 }\end{array}$ & $\begin{array}{c}\text { Imposed Radius of } \\
\text { Curvature (mm) }\end{array}$ & $\begin{array}{c}\text { Superficial } \\
\text { Layer }\end{array}$ & n-value \\
\hline D0 & No & -- & -- & -- & 9 \\
\hline FS & Yes & $20 \%$ FBS & -- & -- & 7 \\
\hline Concave & Yes & $20 \%$ FBS & 3 & Concave & 3 \\
\hline Convex & Yes & $20 \%$ FBS & 3 & Convex & 7 \\
\hline
\end{tabular}

${ }^{\mathrm{b}} \mathrm{FS}$ : 4 days of free swelling

c 20\% FBS: Dulbecco's modified Eagle medium with 20\% fetal bovine serum FBS, ascorbic acid (100 $\mu \mathrm{g} / \mathrm{mL}$ ), antimicrobials (100 U/mL penicillin, $100 \mu \mathrm{g} / \mathrm{mL}$ streptomycin, $0.25 \mu \mathrm{g} / \mathrm{mL}$ Fungizone), amino acids, 0.4 mM L-proline, $2 \mathrm{mM}$ L-glutamine), and 4-(2-hydroxyethyl)-1-piperazineethanesulfonic acid (10 $\mathrm{mM})$ 
Cultured samples were characterized on day 5 as described below and were cut in half in the anterior-posterior direction. One half of each sample was frozen at $-78^{\circ} \mathrm{C}$ until needed for biochemical analysis; the other half was fixed in $4 \%$ paraformaldehyde and stored at $4^{\circ} \mathrm{C}$ in preparation for $\mu \mathrm{CT}$ and qPLM.

\subsection{Characterization}

The sample shape was characterized by the following parameters: thickness, wet weight, and opening angle. Thickness was measured for D0 and FS samples using a noncontact laser micrometer (AccurRange 200 Laser Displacement Sensor, Model AR20012). The recorded thickness was an average of three measurements at various locations on the sample. Wet weight was measured using an analytical balance. The opening angle was previously quantified by the inner opening angle (Figure 9B). However, the cup shaped sample in this experiment meant that a 2D photograph could not capture the inner opening angle. Instead, the outer opening angle was used to characterize the change in shape (Figure 9C). Specifically, three images were taken of the sample disk, rotating it $120^{\circ}$ in between each image (Figure 10). The outer opening angle in each image was found using a custom MATLAB script. The recorded outer opening angle was an average of the three measurements. To address the hypothesis that the cartilage was reshaped into a spherical (or nearly spherical) shape, a coefficient of variation (CV) was calculated for each sample. This was defined by the following equation:

$$
C V=\frac{\text { Standard Deviation of the final measured angles }}{(\text { initial opening angle }- \text { relaxed opening angle) }} \times 100 \%
$$




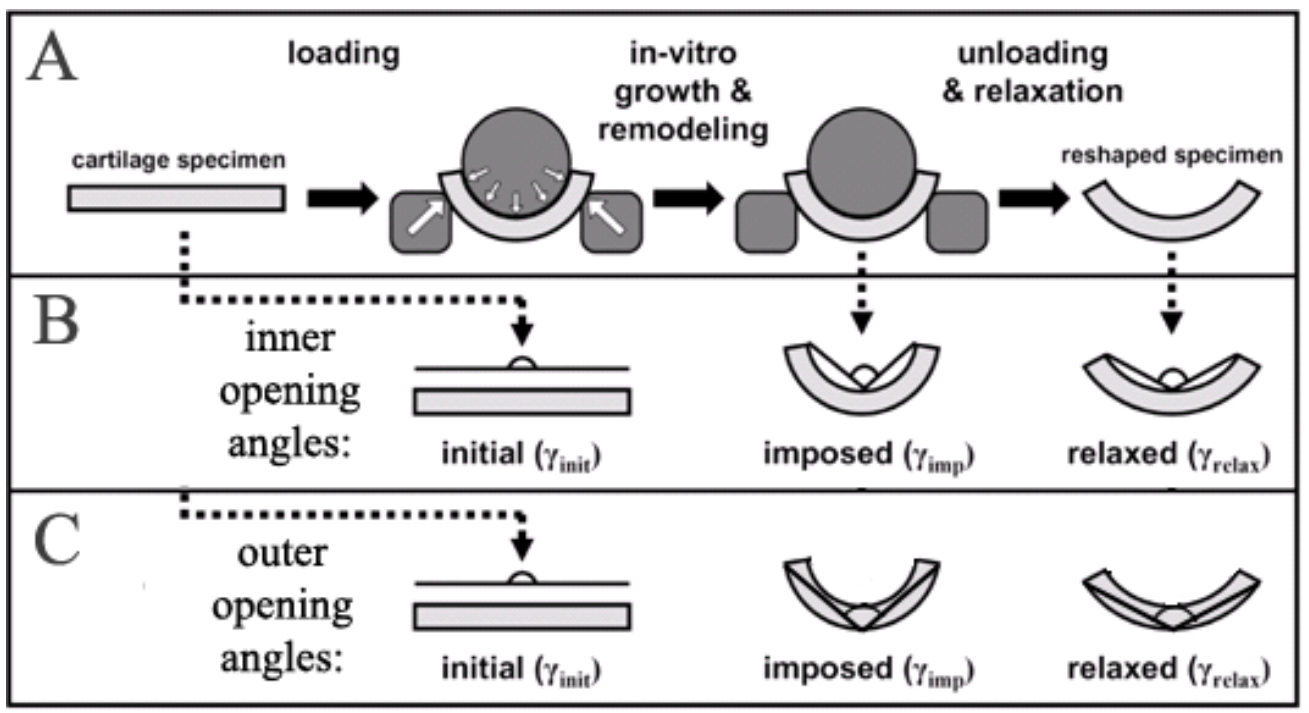

Figure 9: A. Cross sectional loading of cartilage. B. Shape change quantified by inner opening angle $(\gamma)$ as defined previously[26]. C: Shape change quantified by outer opening angle $(\gamma)$. Figure adapted from [26].

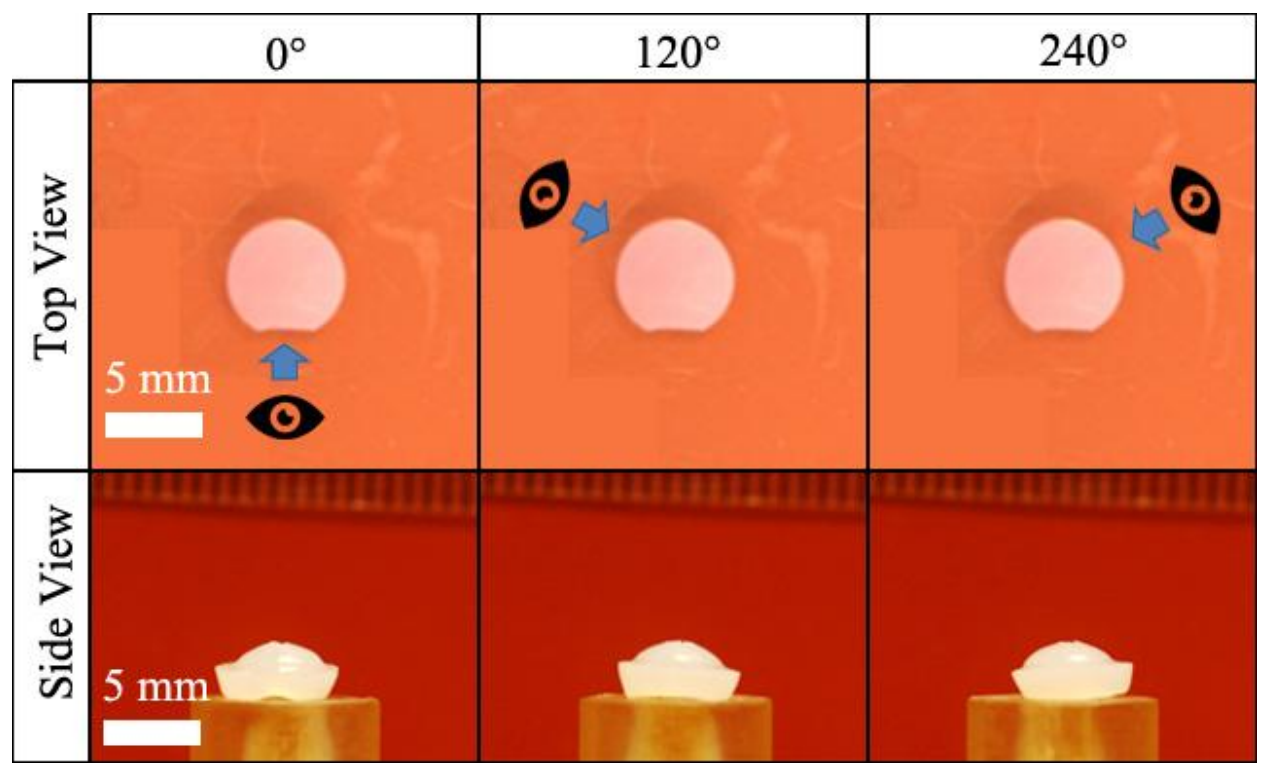

Figure 10: Outer opening angle was calculated as an average of three measurements from pictures equally spaced around the cartilage disk. *Note that in this figure the opening angle is concave down, which is in contrast to Figure 9C that shows the outer opening angle as concave up. 


\subsubsection{Characterization: D0 samples}

The thickness, wet-weight and opening angle of D0 samples were measured on day zero.

\subsubsection{Characterization: FS samples}

The initial thickness, initial wet weight and initial opening angle of the FS samples were measured on day one after the night of free swelling. Final thickness and final wet weight were measured after the samples were removed from culture. Additionally, the relaxed opening angle was recorded 2 hours after the samples were removed from culture.

\subsubsection{Characterization: concave and convex samples}

The initial wet weight and initial opening angle of concave and convex samples were measured on day one after the night of free swelling, but before samples were placed in static spherical bending. The final wet weight was measured after the samples were removed from culture. Additionally, the relaxed opening angle was recorded 2 hours after the samples were removed from culture.

\subsection{Biochemical Analysis}

All biochemical analyses were performed as described below and then normalized to either initial wet weight or final wet weight.

\subsubsection{Biochemical Analysis: Proteinase K (ProK) digestion}

The biochemistry half sample had a $2 \mathrm{~mm}$ half circle punched out of the center of the straight edge. The $2 \mathrm{~mm}$ diameter half circle was weighed wet, lyophilized overnight and weighed dry. The dried samples were solubilized with proteinase $\mathrm{K}(0.5 \mathrm{mg} / \mathrm{mL})$ using a volume equal to $25 \mathrm{x}$ the volume of cartilage and incubated at $60^{\circ} \mathrm{C}$ for more than 
16 hours. Ten $\mu \mathrm{L}$ of the ProK digest was diluted up to 1:40 with deionized (dI) water in duplicate so that each sample had 2 separate 1:40 dilutions. Biochemical analyses were completed in duplicate.

\subsubsection{Biochemical Analysis: Glycosaminoglycan (GAG) Assay}

In a 384-well plate, $10 \mu \mathrm{L}$ of the 1:40 dilution was combined with $90 \mu \mathrm{L}$ of dimethylmethylene blue (DMMB) dye. In the same plate, $10 \mu \mathrm{L}$ of standard solutions at $0,0.25,0.5,0.75,1.0$, and $1.25 \mu \mathrm{g}$ of GAG per well were combined with $90 \mu \mathrm{L}$ DMMB dye. A second degree polynomial was fit to the standard absorbance $\left(A_{520 \mathrm{~nm}}\right)$ versus $\mu \mathrm{g}$ of GAG, and this curve fit was used to obtain the sample quantity of GAG in each well.

Some issues with the assay were as follows: six samples had a high coefficient of variation $(\mathrm{CV}>15 \%)$. One sample (k2p4z1) had not been completely digested after the first assay. To solve this problem, $1 \mu \mathrm{L}$ of stock $(25 \mathrm{mg} / \mathrm{mL})$ ProK solution was added to the original digest solution for this sample (k2p4z1) and allowed to digest overnight as described above. Also one sample (k4p4z1) had an absorbance value that was outside the range of the standards. These seven samples were re-assayed and the re-assayed values were used in the final analysis.

\subsubsection{Biochemical Analysis: Hydroxyproline Assay}

In a $1.5 \mathrm{~mL}$ centrifuge tube $160 \mu \mathrm{L}$ of the $1: 40$ dilutions were hydrolyzed in $6 \mathrm{~N}$ $\mathrm{HCl}$ overnight at $110^{\circ} \mathrm{C}$. The acid was dried off the following night at $80^{\circ} \mathrm{C}$ leaving a small pellet. The pellet was reconstituted in $125 \mu \mathrm{L}$ of $1 \mathrm{x}$ assay buffer (one part stock buffer $^{\mathrm{d}}$ diluted with nine parts $\mathrm{dI}$ water). In a 384 -well plate, $50 \mu \mathrm{L}$ of the reconstituted

${ }^{\mathrm{d}}$ Stock Buffer: 238mM Citric Acid monohydrate, $882 \mathrm{mM}$ SodiumAcetate trihydrate, 192mM Acetic Acid at $\mathrm{pH}=6$. 
sample was combined with $40 \mu \mathrm{L}$ of Chloramine $\mathrm{T}_{\text {reagent }}{ }^{\mathrm{e}}$ and $40 \mu \mathrm{L}$ DMBA reagent ${ }^{\mathrm{f}}$. In the same plate, $50 \mu \mathrm{L}$ of standard solutions at $0,0.25,0.5,1.0,1.5$ and $2.0 \mu \mathrm{g}$ of hydroxyproline per well were combined with $40 \mu \mathrm{L}$ of Chloramine T reagent and $40 \mu \mathrm{L}$ DMBA reagent. The plate was placed on a sponge in a $60^{\circ} \mathrm{C}$ water bath, and the solutions were allowed to react for 15 minutes. The plate was cooled for 5 minutes and dried on a paper towel before reading the absorbance of the plate at $\mathrm{Abs}=560 \mathrm{~nm}$. The reconstituted samples were also run in duplicate (to effectively make four wells per sample). A linear regression was fit to the standard absorbance versus $\mu \mathrm{g}$ of hydroxyproline, and this curve fit was used to obtain the sample quantity of hydroxyproline in each well. Collagen content was calculated using the conversion factor $7.25 \mathrm{~g}$ of COL per $\mathrm{g}$ of hydroxyproline [30,31].

The only issue in this assay was that five samples initially had high CV and were re-assayed. Because there was a limited amount of digest, only $80 \mu \mathrm{L}$ of the 1:40 dilutions were hydrolyzed. Consequently, only two wells were run (one well per hydrolysate). The re-assayed values were used in further analysis.

\subsubsection{Biochemical Analysis: DNA Assay}

In a 96-well plate, $20 \mu \mathrm{L}$ of the 1:40 dilutions were combined with $80 \mu \mathrm{L}$ of $1 \mathrm{x}$ TE, and $100 \mu \mathrm{L}$ of PicoGreen dye. Standards were prepared similarly with $0,2,10,20$, 40, and $100 \mathrm{ng}$ of DNA per well. The fluorescence was recorded with excitation of 480 $\mathrm{nm}$ and emission at $520 \mathrm{~nm}$. A linear regression was fit to the standards and used to

\footnotetext{
${ }^{\text {e }}$ Chloramine T reagent: 50mM Chloramine T, 26\% n-propanol, Stock Buffer

${ }^{\mathrm{f}}$ DMBA (p-dimethylaminobenzaldehyde) reagent: $1 \mathrm{M}$ p-dimethylaminobenzaldehyde, $60 \% \mathrm{n}$-propanol, $16 \%$ perchloric acid.
} 
calculate the DNA content of the digests. Cell content was calculated using the conversion factor $7.7 \mathrm{pg}$ of DNA per cell [32].

Some of the samples in this assay had a low fluorescence and were re-assayed at a more concentrated dilution. Additionally samples with high CV were re-assayed.

\subsection{Micro-computed tomography $(\mu \mathrm{CT})$}

Micro-computed tomography $(\mu \mathrm{CT})$ samples were fixed in $4 \%$ paraformaldehyde for at least 48 hours. Then samples were placed in $20 \%$ Hexabrix $^{\mathrm{g}}$ for at least 24 hours before imaging. On the day of imaging, samples were dried of excess Hexabrix solution by dabbing the sample with a Kim Wipe $®$. The samples were then placed in a $15 \mathrm{~mL}$ Falcon $^{\mathrm{TM}}$ tube between layers of Styrofoam. The tube was loaded into the SkyScan 1076 (SkyScan, Kontich, Belgium) to obtain a 3D image of the sample. The resolution was 9 $\mu \mathrm{m} / \mathrm{voxel}$, a cubic version of a pixel.

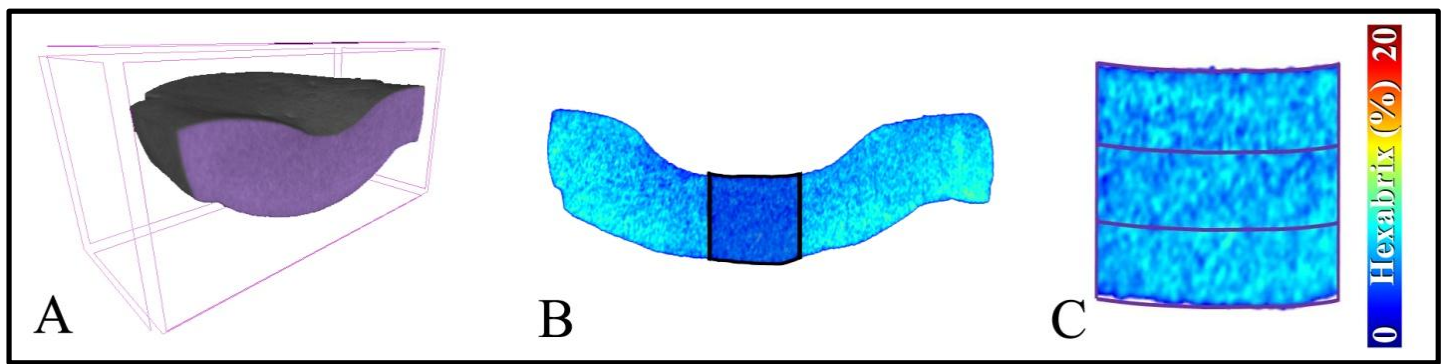

Figure 11: A. Representative 3D image of a Convex sample after imaged by micro CT. Purple represents the slice that was taken to create the image in $B$. The center 119 pixel width (highlighted in darker blue) represents $C$, which has been split into thirds for quantitative analysis.

Using DataViewer ${ }^{\mathrm{TM}}$, a slice was taken from the 3-dimmensional data from the most central part of the sample that was continuous (Figure 11A). Then the slice was analyzed by a custom MATLAB script to quantify the attenuation in the center 119 pixels

g 20\% Hexabrix: Hexabrix 320 (HX-320-100, 100ml bottles, Guerbet, Bloomington, IN) in Phosphate Buffered Saline (PBS) with Proteinase inhibitors (PIs) $(1 \mathrm{mM}$ phenylmethanesulfonyl fluride, $2 \mathrm{mM}$ disodium ethylenediamine tetraacetate, $5 \mathrm{mM}$ benzamidine- $\mathrm{HCl}, 10 \mathrm{mM}$ N-ethylmaleimide) 
of the sample (Figure 11C). The attenuation was converted to a \% Hexabrix concentration at each pixel using a standard curve. Solutions for the standard curve had Hexabrix concentrations of $0,5,10,15$ and 20\% Hexabrix. The \% Hexabrix values provided an inverse relationship to the local concentration of proteoglycans (PG). The data was split into top, middle and bottom thirds for analysis. Even though the superficial layer may appear in the image on the bottom such as in convex samples, it is still considered the top so that comparisons are made as such: superficial-superficial, middlemiddle, and bottom-bottom when comparing across sample groups.

\section{6. qPLM}

\subsection{1. qPLM: Cryosectioning}

After $\mu \mathrm{CT}$, samples were placed again in $4 \%$ paraformaldehyde and stored at $4^{\circ} \mathrm{C}$, for at least 48 hours. Samples were then snap-frozen in OCT (optimum cutting temperature) embedding compound. Using a cryostat, the embedded samples were sliced from the central cross section into $10 \mu \mathrm{m}$ thick sections and placed on either "Superfrost ® Plus Gold" microscope slides or Polylysine microscope slides.

\subsection{2. qPLM: Imaging}

Using PBS, excess OCT compound was rinsed from slides to avoid particles that might interfere with the imaging. The samples were then rehydrated, cover slipped and placed on the microscope stage. The objective was set to $10 \mathrm{x}$ on the polarized light microscope and focused on the center of the sample. The resolution was $1.67 \mu \mathrm{m}$ per pixel. A series of seven angle images were taken with the polarizer-analyzer set to 0-90, $15-105,30-120,45-135,60-150,75-165$, and 90-180. For each angle combination the polarizer-analyzer was in crossed polarizer position, where the image background was as 
dark as possible. Two more images were taken with a quarter wave plate; the polarizer was set to $45^{\circ}$ and the analyzer at $134^{\circ}$ then $136^{\circ}$. This distinguished between $0-90^{\circ}$ and $90-180^{\circ}$. The final image taken was a background image with the polarizer and analyzer at $90^{\circ}$ to one another. The thickness of most samples exceeded the height of the field of view so that two sets of images were stitched together to capture the full thickness of the sample. Images were stitched together and analyzed with a custom MATLAB script to obtain the alpha ( $\alpha$ ), parallelism index (PI), and area fraction of non-birefringent tissue $\left(\mathrm{AF}_{\mathrm{NBR}}\right) . \alpha$ indicates the primary orientation of fibers with respect to the superficial surface normal, where $0^{\circ}$ is horizontal and parallel with the tissue top and bottom surfaces (Figure 12); $\mathrm{PI}$ indicates how aligned the collagen fibers are; $\mathrm{AF}_{\mathrm{NBR}}$ indicates where tissue is highly disorganized or has low birefringence.

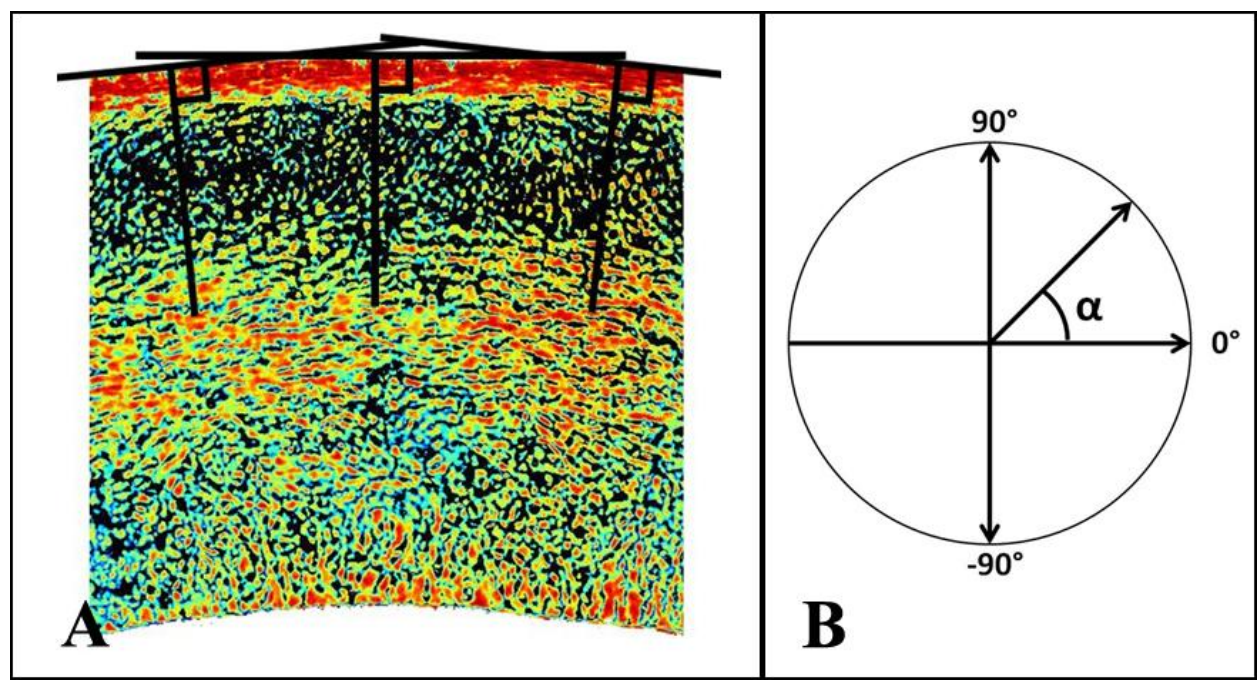

Figure 12: A. Example of measurement to the surface normal. B. The axis used for each individual pixel is in reference to the direction of the line from that pixel to the nearest point on the surface of the tissue.

\subsection{Statistical Comparisons}

Data are presented as means \pm standard deviation. Analysis of variance (ANOVA) with post hoc Tukey testing, when applicable, was used to determine the effect of culture 
on the relaxed opening angle, water percent, percent change in wet weight and biochemical properties.

Two-way-ANOVA was initially used to determine significant changes in qPLM parameters (PI, alpha, and AF) and percent Hexabrix concentration where the factors were group (D0, FS, concave and convex) and depth (superficial, middle and deep). Depth was used as a repeated factor. Subsequently, one-way ANOVA was performed on all groups with post hoc Bonferroni testing, when applicable, to determine the effect of depth on qPLM parameters percent Hexabrix concentration. Then one-way ANOVA with post hoc Tukey testing, when applicable, was performed on each layer with group as the factor. The significance level, $\alpha$, was 0.05 for all tests. 


\section{RESULTS}

\subsection{Geometry}

Articular cartilage spherical bending for 4 days of culture resulted in a cupped tissue shape. Visual conformation of cartilage reshaping is shown in Figure 13. The day 0 (D0) and free swell (FS) samples both have nearly flat surfaces; however, concave and convex samples maintained the bent shape after culture in static bending (Figure 13). Quantification of the shape change revealed that the relaxed opening angle of concave $\left(\gamma_{\text {relax }}=144^{\circ} \pm 9^{\circ}\right)$ and convex $\left(\gamma_{\text {relax }}=152^{\circ} \pm 15^{\circ}\right)$ samples were markedly reduced compared with either D0 $\left(\gamma_{\text {relax }}=176^{\circ} \pm 5^{\circ}\right)$ or FS $\left(\gamma_{\text {relax }}=177^{\circ} \pm 8^{\circ}\right)$ controls $(\mathrm{p}<0.001$; Figure 14A). The average coefficient of variation (CV) for the concave samples was 7.5\%. The average $\mathrm{CV}$ for the convex samples was $160 \%$. If we treat $\mathrm{k} 6 \mathrm{p} 1 \mathrm{x} 1$ as an outlier (because one of the opening angle measurements was much greater that $180^{\circ}$ ) and throw it out, the new average CV for convex samples is $15.3 \%$.

The wet weight significantly increased from values on day zero in FS and convex samples $(\mathrm{p}<0.05)$. However, the increase in wet weight was significantly less in convex $(6.5 \% \pm 5 \%)$ samples than in FS $(16 \% \pm 7 \%)$ samples $(\mathrm{p}<0.05$; Figure $14 \mathrm{~B})$. Concave samples had a similar wet weight to convex samples $(\mathrm{p}=0.999)$. Additionally, wet weights for concave and FS were not significantly different $(\mathrm{p}=0.12)$. No significant changes in water content were observed (Figure 14C). 


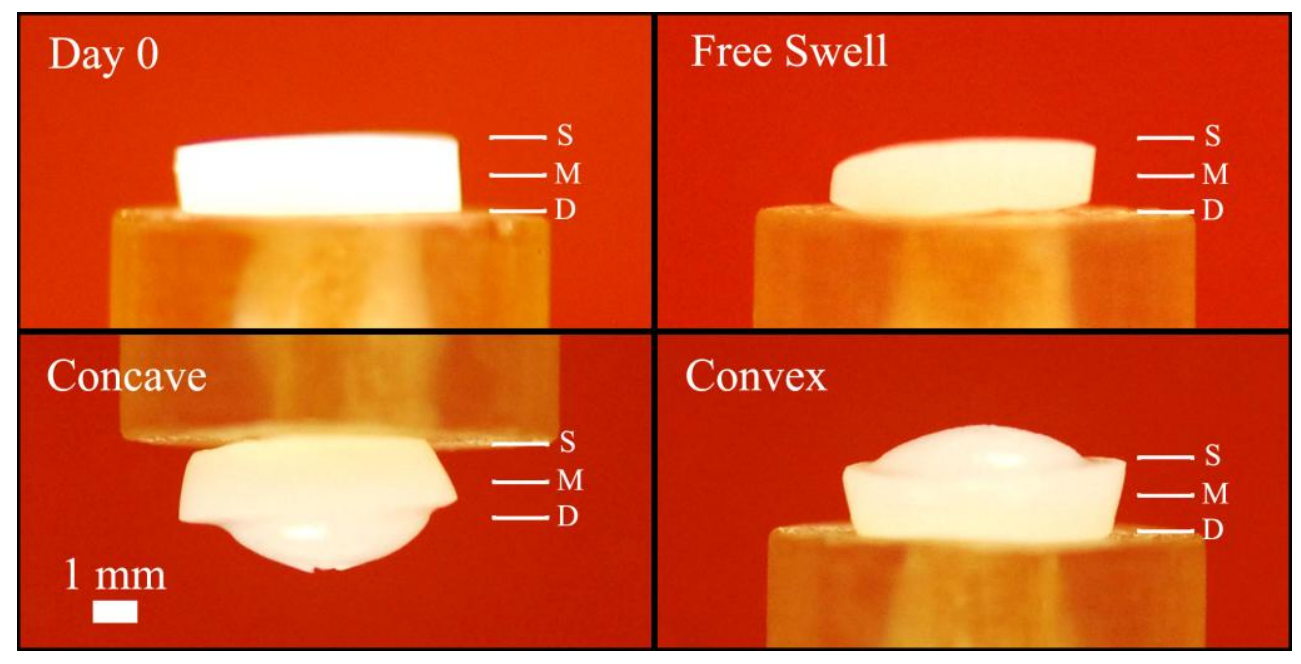

Figure 13: Representative images of samples in the relaxed state from each group. Note the distinct change in shape in Concave and Convex samples when compared to the unbent Day 0 and Free Swell samples. S, M and D indicate superficial, middle and deep layers, respectively. 


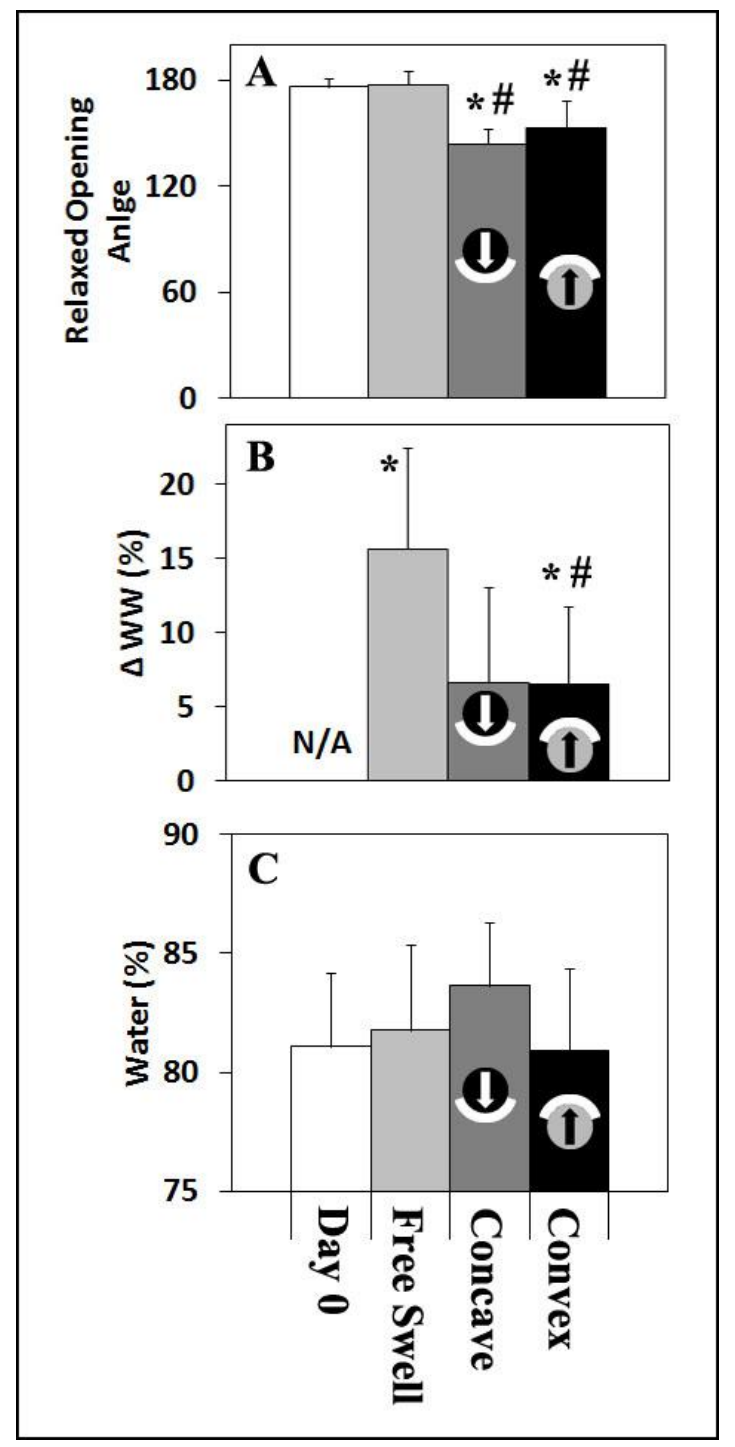

Figure 14: A. Relaxed opening angle $\left(\gamma_{\text {relax }}\right)$ of Day 0 and cultured AC explants. B. Change in wet weight $(\Delta \mathrm{WW})$ of cultured explants. $C$. Water content of $D 0$ and cultured explants. Symbols indicate $\mathbf{p}<0.05$ versus *Day 0 and \#Free Swell. Data is shown as mean \pm SD. D0 $(n=9)$, Free Swell $(n=7)$, Concave $(n=3)$, and Convex $(n=7)$. Symbol inside concave and convex bars indicate which way the cartilage disk was bent with superficial side always up.

\subsection{Biochemistry}

No differences were found for GAG, COL, or cell contents normalized by either initial or final wet weights (Figure 15). 


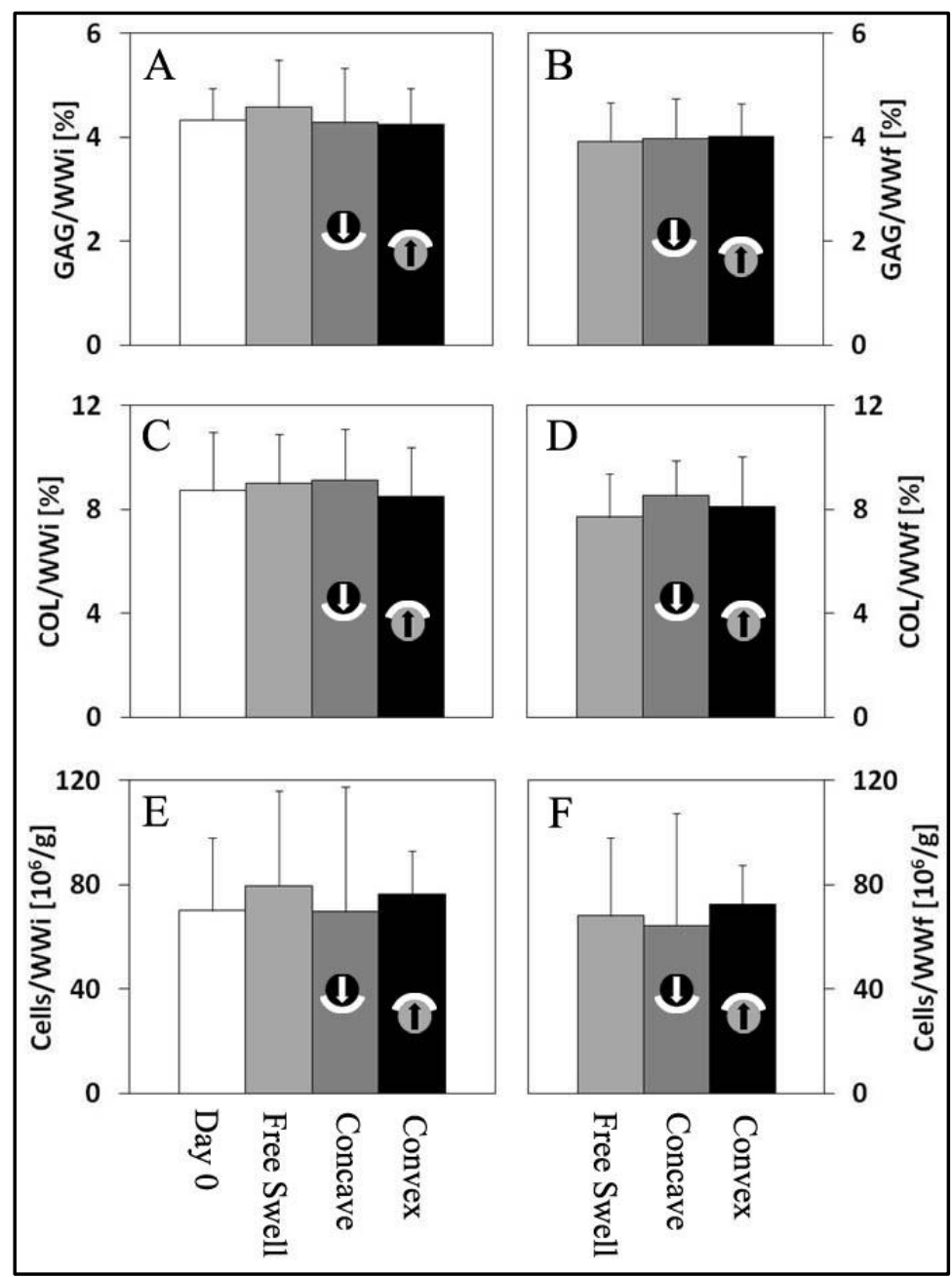

Figure 15: Glycosaminoglycan (GAG) content (A, B); collagen (COL) content $(C, D)$; and cell content $(E, F)$ of Day 0 and cultured explants normalized to initial wet weight (WWi; A, C, E), and final wet weight (WWf; B, D, F). For Day 0, WWi = WWf. Data is shown as mean \pm SD. Day0 $(n=8)$, Free Swell $(n=6)$, Concave $(n=3)$, Convex $(n=6)$.

\subsection{Micro Computed Tomography}

Figure 16 shows that all groups trended to have a decreasing Hexabrix concentration with depth from the articular surface. Comparisons of similar layers (e.g. only the S-layer of each group) by 1-way ANOVA showed significant differences in the deep layer (Table 3). Post hoc Tukey tests revealed that the Hexabrix concentration was significantly lower in the deep layer of convex samples than the deep layer of D0 samples $(\mathrm{p}<0.05)$. Comparisons within groups by 1-way ANOVA revealed significant differences 
in the D0 group and the convex group (Table 4). Post hoc Bonferroni tests revealed that in D0 samples the deep layer had significantly less Hexabrix than the superficial layer $(\mathrm{p}<0.05)$. Also all layers of the convex group were significantly different (SvM: $p<0.01$; SvD: $\mathrm{p}<0.001 ; \mathrm{MvD}: \mathrm{p}<0.001)$.

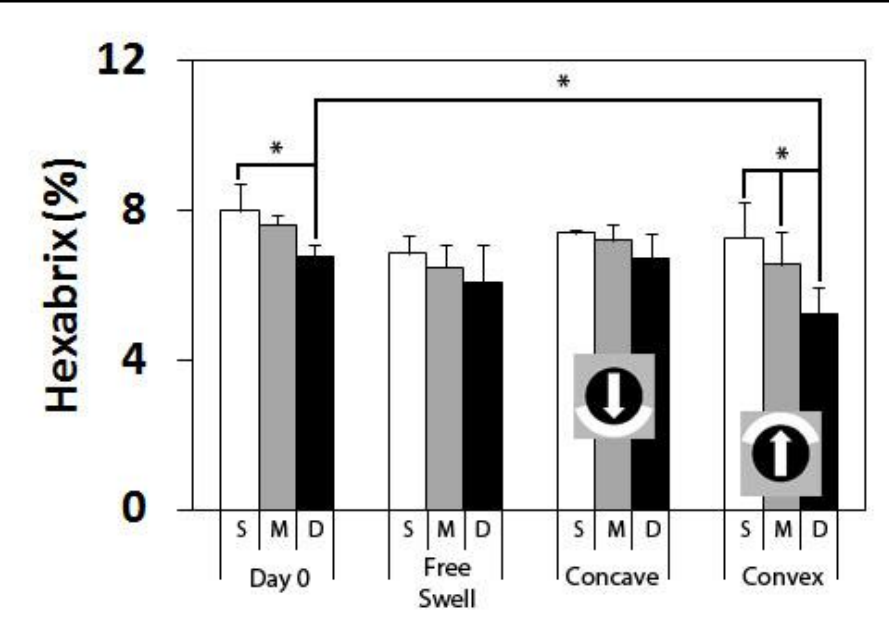

Figure 16: Hexabrix concentration for sample groups Day $0(n=4)$, Free Swell $(n=3)$, Concave $(n=2)$ and Convex $(n=5)$. Each group is split into 3 layers: superficial (S), middle (M) and deep (D). Data is shown as mean \pm SD. * indicates significant differences between linked bars.

Table 3: p-values for 1-way ANOVA Hexabrix concentration (\%) comparing groups. Significant $p$-values $(\mathbf{p}<0.05)$ are shown in BOLD.

\begin{tabular}{|l|c|}
\hline & Hexabrix (\%) \\
\hline S-layer & 0.298 \\
\hline M- layer & 0.114 \\
\hline D-layer & $\mathbf{0 . 0 3 4}$ \\
\hline
\end{tabular}

Table 4: p-values for 1-way ANOVA Hexabrix concentration (\%) comparing layers within each group and using layers as a repeated measure. Significant $p$-values $(\mathbf{p}<\mathbf{0 . 0 5})$ are shown in BOLD.

\begin{tabular}{|l|c|}
\hline & Hexabrix (\%) \\
\hline \hline Day 0 & $\mathbf{0 . 0 1 6}$ \\
\hline Free Swell & 0.078 \\
\hline Concave & 0.375 \\
\hline Convex & $\mathbf{0 . 0 0 0}$ \\
\hline
\end{tabular}




\subsection{Quantitative Polarized Light Microscopy}

Quantitative polarized light microscopy (qPLM) data was recorded at each pixel in the sample image and then grouped into superficial (S), middle (M) or deep (D) layers for analysis. It should be noted that the sections analyzed for $\mathrm{qPLM}$ are close to the $\mu \mathrm{CT}$ section but not exactly the same slice.

\subsubsection{Alpha}

Comparisons of collagen fiber orientation $(\alpha)$ across groups of similar layers showed no significant differences after 1-way ANOVA (Table 5). Comparisons within groups using layers as a repeated measure showed no significant differences either (Table 6). However, convex samples trended toward higher $\alpha$ values in the deep layer (Figure 17A). Comparisons of collagen fiber orientation standard deviation ( $\alpha \mathrm{SD})$ across groups of similar layers did not show any significant differences (Table 5). Comparisons of layers within each group showed that only concave samples had significant differences (Table 6). Post hoc Bonferroni tests revealed that only the $\mathrm{S}$ and $\mathrm{D}$ layers were significantly different $(\mathrm{p}<0.05$; Figure 17B). One trend that is interesting to note is the relatively uniform orientation standard deviation $(\alpha \mathrm{SD})$ in the unbent groups (D0 and FS), yet the concave and convex groups show opposite trends with respect to depth. The concave $\alpha \mathrm{SD}$ decreases with depth, while the convex $\alpha \mathrm{SD}$ increases with depth (Figure 17). 


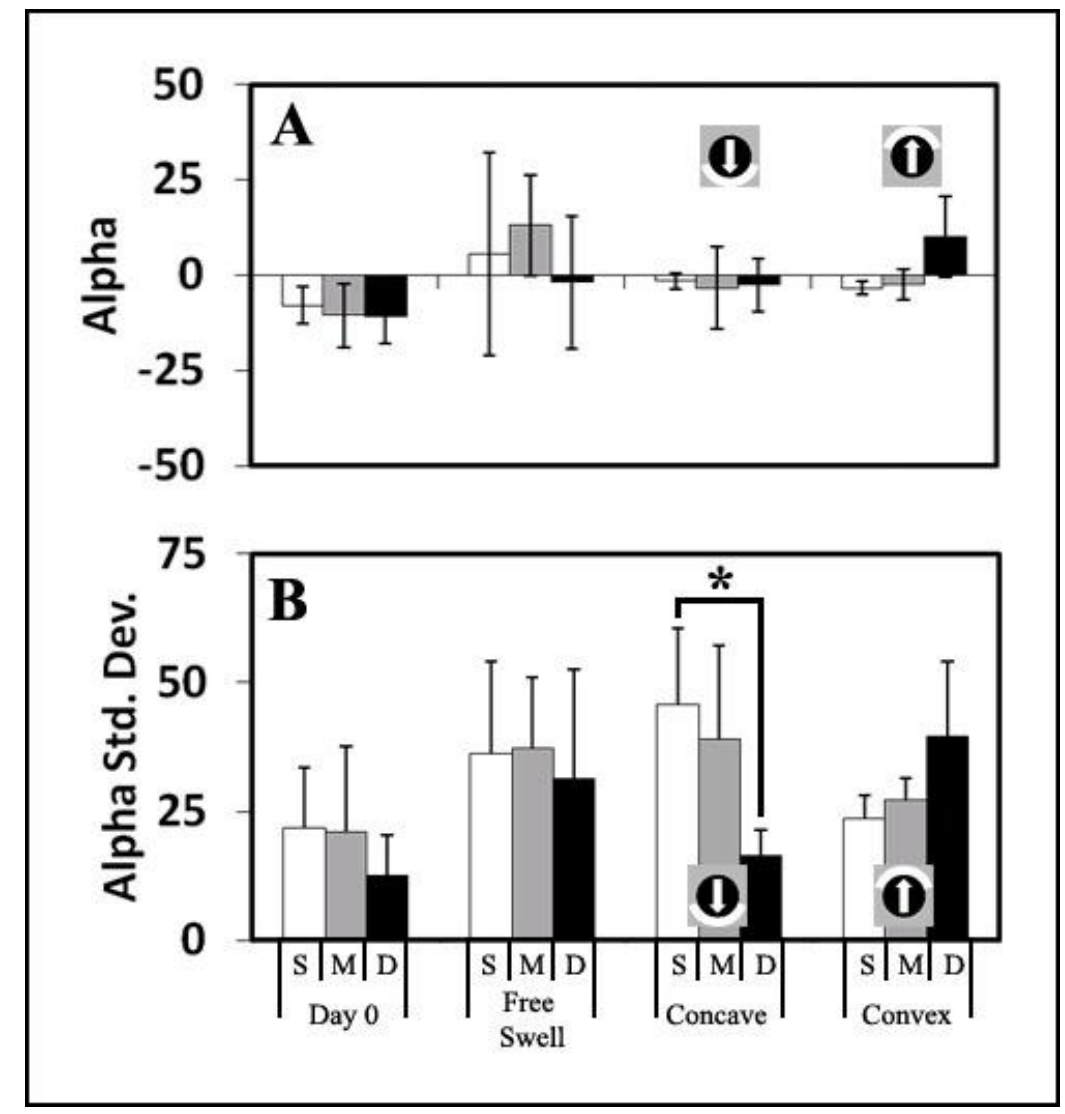

Figure 17: Orientation $\alpha$ (A) and standard deviation of orientation $\alpha$ SD (B) for sample groups Day $0(n=2)$, Free Swell $(n=3)$, Concave $(n=3)$ and Convex $(n=3)$. Data is shown as mean \pm SD. * indicates significant differences between linked bars.

Table 5: p-values for 1-way ANOVA for collagen fiber orientation ( $\alpha$ ) and collagen fiber orientation standard deviation ( $\alpha \mathrm{SD})$ comparing groups

\begin{tabular}{|l|c|c|}
\hline & $\alpha$ & $\alpha \mathrm{SD}$ \\
\hline \hline S-layer & 0.768 & 0.217 \\
\hline M- layer & 0.127 & 0.464 \\
\hline D-layer & 0.327 & 0.193 \\
\hline
\end{tabular}

Table 6: $p$-values for 1-way ANOVA for $\alpha$ and $\alpha$ SD comparing layers within each group and using layers as a repeated measure. Significant $p$-values $(p<0.05)$ are shown in BOLD.

\begin{tabular}{|l|c|c|}
\hline & $\alpha$ & $\alpha$ SD \\
\hline \hline Day 0 & 0.333 & 0.269 \\
\hline Free Swell & 0.724 & 0.859 \\
\hline Concave & 0.884 & $\mathbf{0 . 0 2 1}$ \\
\hline Convex & 0.060 & 0.226 \\
\hline
\end{tabular}




\subsubsection{Parallelism Index}

Parallelism index (PI) comparisons of similar layers (e.g. S-layer) across groups by ANOVA showed no significant differences (Table 7). Analysis by 1-way ANOVA on each group with layers as a repeated measure showed that only concave samples had a significant difference among layers (Table 8). However, upon post hoc Bonferroni testing no significant differences were shown, yet comparison between $\mathrm{S}$ and $\mathrm{D}$ layers of concave samples was nearly significant with $p=0.0554$. Another interesting point is that the D0, FS and concave groups all trended the same way with PI increasing with depth (Figure 18A). The only non-conforming group was convex, where the deep layer did not display the expected increase in PI over the other two layers.

Parallelism index standard deviation (PISD) comparisons by 1-way ANOVA showed no significant differences between groups of similar layers (Table 7). Analysis by 1-way ANOVA within groups using layers as a repeated measure showed that only FS samples had significant differences (Table 8). Furthermore, Bonferroni post hoc analysis showed that the S and D layers of the FS group were significantly different $(\mathrm{p}<0.05)$. It is also interesting to note the decreasing PISD in D0, FS and convex groups (Figure 18B). The only non-conforming group was concave where the D-layer did not display the expected decrease in PISD that was found in the other groups (Figure 18B). 


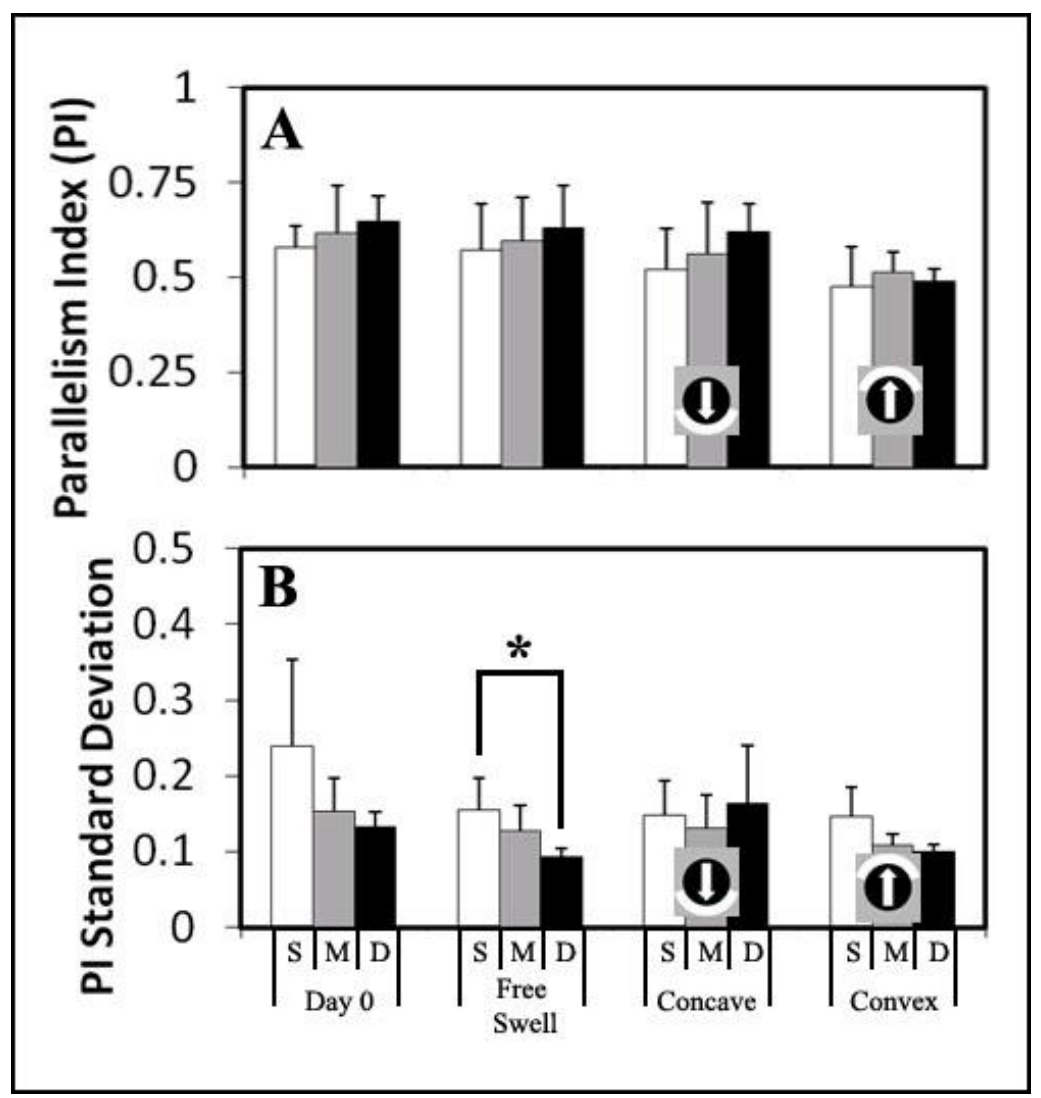

Figure 18: Parallelism index (A) and standard deviation of parallelism index (B) for sample groups Day 0, Free Swell, Concave and Convex ( $n=3$ for all groups). Data is shown as mean \pm SD. $*$ indicates significant differences between linked bars.

Table 7: p-values for 1-way ANOVA for Parallelism Index (PI) and Parallelism Index Standard Deviation (PISD) comparing groups

\begin{tabular}{|l|c|c|}
\hline & PI & PI SD \\
\hline \hline S-layer & 0.582 & 0.350 \\
\hline M- layer & 0.700 & 0.538 \\
\hline D-layer & 0.117 & 0.190 \\
\hline
\end{tabular}

Table 8: p-values for 1-way ANOVA for PI and PISD comparing layers within each group and using layers as a repeated measure. Significant $p$-values $(p<0.05)$ are shown in BOLD.

\begin{tabular}{|l|c|c|}
\hline & PI & PI SD \\
\hline Day 0 & 0.301 & 0.239 \\
\hline Free Swell & 0.763 & $\mathbf{0 . 0 3 3}$ \\
\hline Concave & $\mathbf{0 . 0 4 5}$ & 0.370 \\
\hline Convex & 0.748 & 0.065 \\
\hline
\end{tabular}




\subsubsection{Area Fraction}

Area fraction (AF) comparisons of similar layers and across groups showed no significant differences (Table 9). However, comparisons within groups using layers as a repeated measure revealed significant differences within the D0 group and concave groups. Post hoc Bonferroni tests revealed that the D layer was significantly higher than the M layer $(\mathrm{p}<0.01)$ and $\mathrm{S}-$ layer $(\mathrm{p}<0.005)$ in the D0 group. Additionally, the D layer was significantly higher than the $S$ layer in the concave group $(\mathrm{p}<0.05)$. In all groups the AF increased with depth (Figure 19A).

Comparisons of area fraction standard deviation (AFSD) of similar layers across groups showed no significant differences (Table 9). Comparisons within groups using layers as a repeated measure revealed significant differences in the D0 group (Table 10). Post hoc Bonferroni tests further showed that AFSD was significantly lower in the D layer than the S layer of D0 samples $(\mathrm{p}<0.05)$. While there was a slight decrease in AFSD with depth, the trend was not as clear as some of the other measurements (Figure 19B). 


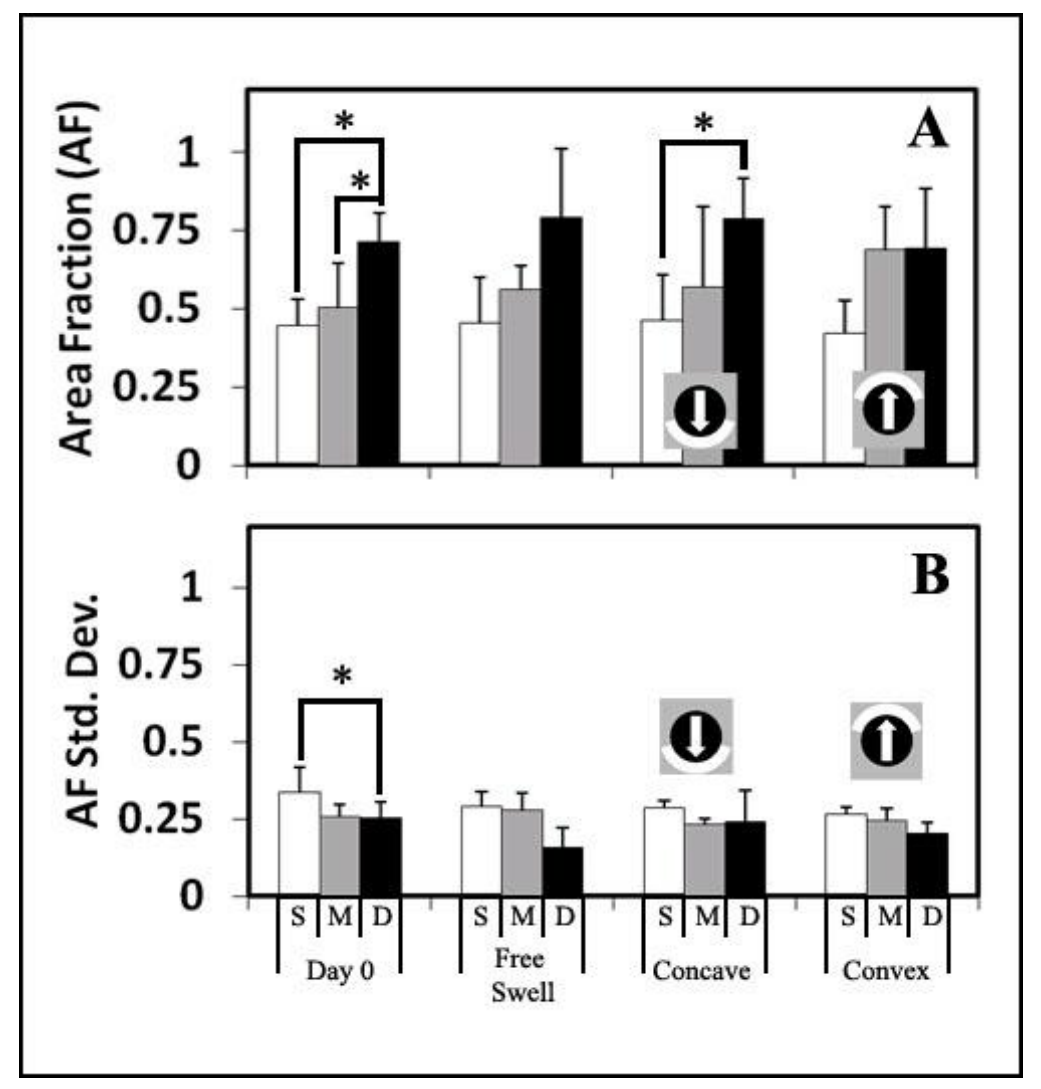

Figure 19: Area Fraction (AF) (A) and Standard Deviation of Area Fraction (B) for sample groups Day 0, Free Swell, Concave, and Convex ( $n=3$ for all groups). Data is shown as mean \pm SD. $*$ indicates significant differences $(p<0.05)$ between linked bars using a post hoc Bonferroni test that accounts for repeated measures.

Table 9: p-values for 1-way ANOVA for area fraction (AF) and area fraction standard deviation (AFSD) comparing groups

\begin{tabular}{|l|c|c|}
\hline & AF & AF SD \\
\hline \hline S-layer & 0.973 & 0.462 \\
\hline M- layer & 0.598 & 0.591 \\
\hline D-layer & 0.838 & 0.353 \\
\hline
\end{tabular}

Table 10: p-values for 1-way ANOVA for AF and AFSD comparing layers within each group and using layers as a repeated measure. Significant $p$-values $(p<0.05)$ are shown in BOLD.

\begin{tabular}{|l|c|c|}
\hline & AF & AF SD \\
\hline Day 0 & $\mathbf{0 . 0 0 2}$ & $\mathbf{0 . 0 2 6}$ \\
\hline Free Swell & 0.186 & 0.083 \\
\hline Concave & $\mathbf{0 . 0 1 4}$ & 0.603 \\
\hline Convex & 0.163 & 0.074 \\
\hline
\end{tabular}




\section{DISCUSSION}

\subsection{Main Conclusions}

Four days of spherical bending with $20 \%$ FBS significantly reshapes an articular cartilage disk into a cup shape. This change in shape was quantified by outer opening angle and was visually apparent. Furthermore, concave and convex specimens reshaped to nearly the same degree when quantified by outer opening angle $\left(\gamma_{\mathrm{rel}}=143^{\circ}\right.$ for concave specimens, and $\gamma_{\mathrm{rel}}=153^{\circ}$ for convex specimens). The creation of a spherical (or nearly spherical) shape was confirmed in the low $\mathrm{CV}$ between the 3 measurements on each sample (average CV of $7.5 \%$ for concave and $15.3 \%$ for convex). Four types of microstructural changes were measured to assess which were candidates for the shaping, PG, PI (mean and SD), $\alpha$ (mean and SD), and AF (mean and SD). The most promising candidate that may account for microstructural changes is $\alpha \mathrm{SD}$.

Mean orientation $(\alpha)$ of collagen fibers was near $0^{\circ}$ for all groups. This is expected for immature cartilage which does not have the same zonal architecture as adult tissue [5]. The more interesting result is the variation in collagen fiber orientation indicated by orientation standard deviation $(\alpha S D)$. For both D0 and FS groups the variation was about equal throughout the depth. However, concave and convex specimens displayed opposite trends with regards to $\alpha \mathrm{SD}$. In concave specimens, $\alpha \mathrm{SD}$ decreased with depth. Conversely, in convex specimens, aSD increased with depth. This suggests that fibers on the compression side become more scattered and/or less oriented.

The distribution of proteoglycans was significantly different between layers of D0 and convex samples. However, trends appeared to follow the assumption that proteoglycans are mostly immobile in the cartilage matrix. Therefore we reject the 
hypothesis that proteoglycan redistribution accompanies spherical shaping of articular cartilage. At the very least the redistribution is minimal.

\subsection{Interpretation of Results}

Wet weight increased for all cultured specimens. This is consistent with the fact that FBS induces tissue swelling [26]. FS samples increased in wet weight by $15.6 \%$, while concave and convex samples only increased in wet weight by $\sim 6.5 \%$ indicating that spherical bending may have restricted tissue swelling.

There were no significant differences in the water content, which remained in the normal range for immature bovine tissue [33]. No significant differences were observed in the average concentration of collagen, proteoglycan or cell content. This is consistent with uniaxial bending experiments [26], and supports the hypothesis that remodeling of the matrix constituents accompany cartilage reshaping.

Hexabrix concentration decreased with increasing depth in all sample groups. Hexabrix concentration is inversely proportional to the concentration of proteoglycans. Therefore, it is indicated that the proteoglycan concentration increases with depth from the articular surface in all sample groups. D0 samples display the decrease in Hexabrix with depth (consequently an increase in proteoglycans with depth) that has been observed previously [7]. Results show that the decrease in Hexabrix from articular surface to the deep layer is most drastic in convex samples. In contrast, the concave samples show the most uniform concentration of Hexabrix across the depth. These observations may become clear under the hypothesis that the proteoglycans are considered to be immobile in the extracellular matrix [34]. In this context, regions of compression are associated with an increase in proteoglycan concentration (decrease in Hexabrix) when compared 
with control groups (Figure 20). The increase in proteoglycan concentration is then assumed to be due to the fact that compressive strains hold the proteoglycans close together. Conversely, on the tensile side of the bent cartilage, proteoglycans are separated. This would explain the variation trends of concave and convex specimens.

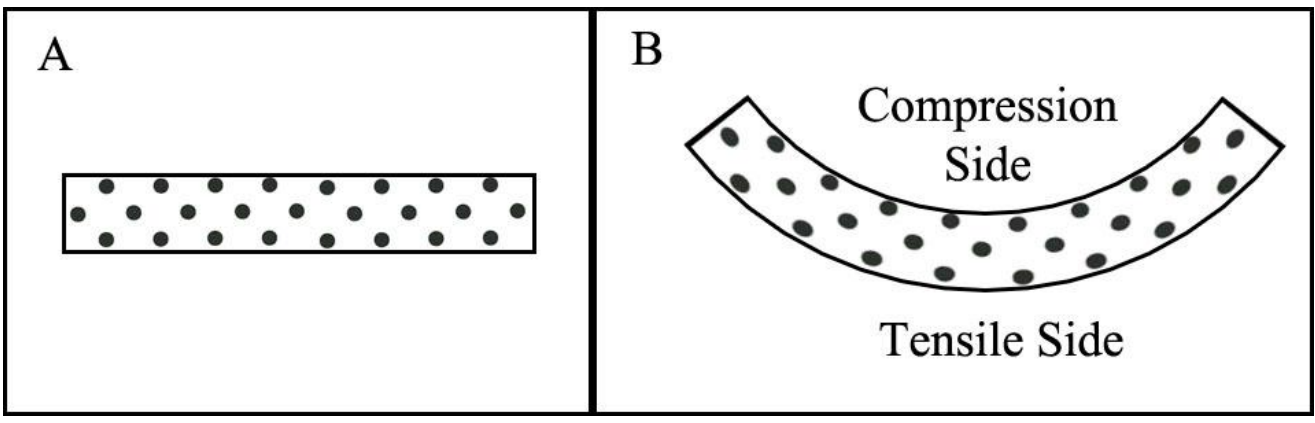

Figure 20: A. Sample before bending B. Sample after bending. Dots represent proteoglycans which become closer together on the compression side and farther apart on the tensile side. Proteoglycans may not necessarily redistribute yet apparent changes in local concentration may appear.

Parallelism index (PI) was similar for D0 and FS controls, yet it appears to decrease overall in the concave and convex specimens. One hypothesis for this may be that the asymmetric strain distribution causes a majority of the tissue to be in compression. For uniaxial bending nearly $75 \%$ of the tissue depth is in compression (Figure 21; [35]). Therefore all three layers of concave and convex specimens are in part subjected to compression accompanied by matrix disorganization. While not significant, PI increases with depth in D0, FS and concave samples. Conversely, convex specimens did not maintain this same trend. Again this continues to support the conclusion above that collagen fibers on the compressive side become more randomly oriented. 


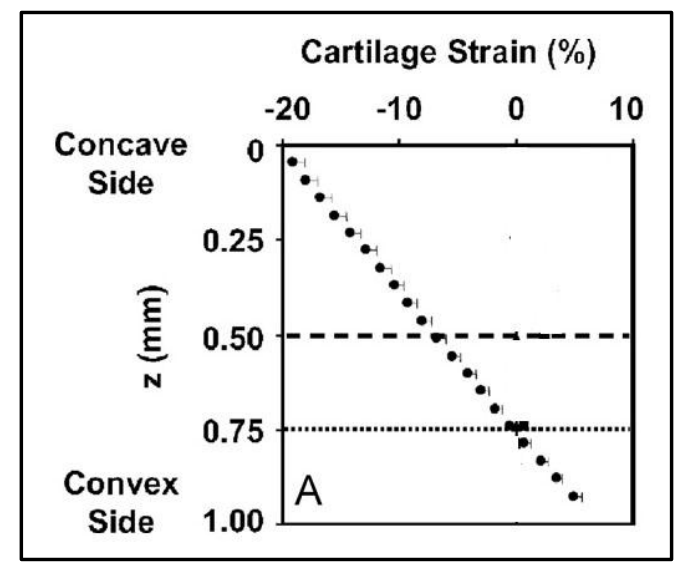

Figure 21: Asymmetric strain distribution of articular cartilage in static uniaxial bending. Dashed line indicates midline of tissue sample. Dotted line indicates the neutral axis. Figure from [35].

The area fraction of matrix constituents can be difficult to interpret because it is the total area of the tissue minus the area of the non-birefringent tissue. The nonbirefringent tissue may represent highly cellular areas or areas where there is little or no collagen. When comparing results from other studies it is evident that low area fraction corresponds with areas of high cellularity in the superficial region of the cartilage tissue [8]. The more distinguished profile of cellularity would most likely be mimicked in the area fraction if the analysis was done in more layers.

\subsection{Limitations}

There were two main aims of this study: 1) To produce significant spherical bending of articular cartilage explants and 2) as a pilot study, to identify microstructural parameters for future in depth analysis. These aims were successful, yet there were some limitations that should be considered in future studies. First, the sample size for the qPLM and $\mu \mathrm{CT}$ analysis only had at most $\mathrm{n}=5$. This greatly limited the statistical power in finding specifically where significant differences lie. For instance, in the $\mu \mathrm{CT}$ analysis there was a consistent trend in all groups of decreasing Hexabrix concentration with depth, yet only the D0 and convex groups showed significant differences. This is most 
likely because these groups had $n=4$ and $n=5$ respectively, while the FS group only had $\mathrm{n}=3$ and concave only had $\mathrm{n}=2$. Similar problems occurred for the qPLM parameters.

One of the major problems related to the application of this experiment is that there is no consideration for integration using the subchondral bone. Most good cartilage autografts or allografts have subchondral bone attached to aid in donor cartilage integration into the recipient [36]. Additionally, static bending of full thickness cartilage explants would most likely fracture the subchondral bone or fracture the cartilage tissue due to the difference in mechanical properties. One solution may be to induce a calcified region after the reshaping of the cartilage tissue. A recent study shows that culture with $\beta$ glycerophosphate induces calcification only in the deep zone of articular cartilage [37]. In this way cartilage explants could be reshaped and then provided with a calcified region for proper integration into the defect site.

Another issue may be that the distribution of collagen fiber content was not mapped in this experiment. It would be useful to know if the local concentration of collagen changes for a specific treatment. This could have been done with infrared imaging [7]; however, it should be noted that the area fraction is a similar measurement, but also includes areas of low birefringence and/or high cellularity. Similarly, it may have been useful to map cell distributions with picogreen staining, but previous results with uniaxial bending specimens have indicated that darkfield imaging of cell lacunae may provide information as well. If it were determined that this study needed to track cellular distribution, it may be possible to use images that were taken with dark field microscopy to give an estimate of the cellular distribution [38]. 
Changes in wet weight were measured using the original $6 \mathrm{~mm}$ sample, whereas the biochemical properties (water \%, collagen, proteoglycans and cells) are based only on the center $2 \mathrm{~mm}$ diameter of the sample. In a future study, it may be interesting to observe how much cartilage wet weight increases only in regions of maximum bending similar to the measurements of the biochemical properties.

\subsection{Application}

This research has implications in tissue engineering of shaped autologous grafts for patients with arthritis. For large cartilage defects it may be useful to reshape autologous tissue into the proper shape using static bending. This study specifically applies to spherical joints such as the humoral head in the shoulder, the femoral head in the hip or the femoral condyles in the knee, which are all bent in multiple directions. Static bending of articular cartilage ensures that the cells remain alive when compared to other reshaping mechanisms [26]. Additionally it models the native microstructural architecture of cartilage tissue much more accurately than other types of grafts such as constructs.

\subsection{Future work}

Future work should include increasing the sample size in the $\mu \mathrm{CT}$ and qPLM analyses. Even though several significant differences are already apparent, a larger sample size would help to solidify any trends that are evident by data means but that are not necessarily significant. Also it would be useful to repeat this experiment with adult tissue because previous studies have shown that calf and adult tissue behave differently under static bending [39]. The adult tissue would have a more defined architecture. With the goal in mind that this research could be used to reshape autologous tissue, most 
patients with arthritis are adults who would have well defined architectural microstructure in their articular cartilage [9]. Therefore adult tissue would more accurately represent the population in need.

Another interesting study may be to look at extended time points with static bending to observe any further microstructural changes that may occur in the tissue. This would be based on the hypothesis that the observed alteration in microstructure at 4 days of culture is an intermediate step in reorganization, and that alterations in microstructure have not yet reached equilibrium.

Finally, it would be useful to split the matrix analysis into more than just three layers. This would more accurately represent the distribution of matrix constituents along the depth of the tissue. The data for this is available, yet for simplicity, this study has been restricted to observation of samples analyzed in only 3 layers.

\subsection{Conclusion}

In vitro spherical bending reshapes immature bovine cartilage into a "cup" shape. Reshaping via spherical static bending is accompanied by matrix remodeling of the collagen network. Collagen fibers associated with areas of compression became more isotropic and disorganized as indicated by the orientation standard deviation. Conversely, collagen fibers associated with areas of tension remained anisotropic. 


\section{REFERENCES}

1. Mow, V.C. and W.C. Hayes, Basic orthopaedic biomechanics. 2nd ed1997, Philadelphia: Lippincott-Raven. xiii, 514 p.

2. Bartel, D.L., D.T. Davy, and T.M. Keaveny, Orthopaedic biomechanics : mechanics and design in musculoskeletal systems. Pearson Prentice Hall bioengineering2006, Upper Saddle River, N.J.: Pearson/Prentice Hall. x, 370 p.

3. Martin, R.B., D.B. Burr, and N.A. Sharkey, Skeletal tissue mechanics1998, New York: Springer. xiv, $392 \mathrm{p}$.

4. Stockwell, R.A., The interrelationship of cell density and cartilage thickness in mammalian articular cartilage. Journal of anatomy, 1971. 109(Pt 3): p. 411-21.

5. Julkunen, P., et al., Maturation of collagen fibril network structure in tibial and femoral cartilage of rabbits. Osteoarthritis and cartilage / OARS, Osteoarthritis Research Society, 2010. 18(3): p. 406-15.

6. Nordin, M. and V.H. Frankel, Basic biomechanics of the musculoskeletal system. 3rd ed2001, Philadelphia: Lippincott Williams \& Wilkins. xvii, 467 p.

7. Rieppo, J., et al., Spatial Determination of Water, Collagen and Proteoglycan Contents by Fourier Transformed Infrared Imaging and Digital Densitometry, in 50th Annual Meeting of the Orthopaedic Research Society.

8. Jadin, K.D., et al., Depth-varying density and organization of chondrocytes in immature and mature bovine articular cartilage assessed by $3 d$ imaging and analysis. The journal of histochemistry and cytochemistry : official journal of the Histochemistry Society, 2005. 53(9): p. 1109-19.

9. Arthritis: Meeting the Challenge of Living Well at a Glance 2012, 2012, Center for Disease Control and Prevention.

10. YJ Cheng MD, P., Prevalence of Doctor-Diagnosed Arthritis and ArthritisAttributable Activity Limitation, 2010.

11. Osteoarthritis. 2011 [cited 201329 April 2013]; Available from: http://www.cdc.gov/arthritis/basics/osteoarthritis.htm.

12. Sofat, N., V. Ejindu, and P. Kiely, What makes osteoarthritis painful? The evidence for local and central pain processing. Rheumatology, 2011. 50(12): p. 2157-65.

13. Setton, L.A., et al., Altered structure-function relationships for articular cartilage in human osteoarthritis and an experimental canine model. Agents and actions. Supplements, 1993. 39: p. 27-48.

14. Bank, R.A., et al., The increased swelling and instantaneous deformation of osteoarthritic cartilage is highly correlated with collagen degradation. Arthritis and rheumatism, 2000. 43(10): p. 2202-10.

15. Caligaris, M., et al., Investigation of the frictional response of osteoarthritic human tibiofemoral joints and the potential beneficial tribological effect of healthy synovial fluid. Osteoarthritis and cartilage / OARS, Osteoarthritis Research Society, 2009. 17(10): p. 1327-32.

16. Ray, C.D. Osteoarthritis Symptoms. [cited 20132 May 2013]; Available from: http://www.spine-health.com/conditions/arthritis/osteoarthritis-symptoms.

17. Steadman, J.R., W.G. Rodkey, and K.K. Briggs, Microfracture to treat fullthickness chondral defects: surgical technique, rehabilitation, and outcomes. The journal of knee surgery, 2002. 15(3): p. 170-6. 
18. Brittberg, M., Autologous chondrocyte transplantation. Clinical orthopaedics and related research, 1999(367 Suppl): p. S147-55.

19. Hangody, L., et al., Mosaicplasty for the treatment of articular defects of the knee and ankle. Clinical orthopaedics and related research, 2001(391 Suppl): p. S32836.

20. Bugbee, W.D. and F.R. Convery, Osteochondral allograft transplantation. Clinics in sports medicine, 1999. 18(1): p. 67-75.

21. Williams, G.M., et al., Shape, loading, and motion in the bioengineering design, fabrication, and testing of personalized synovial joints. Journal of biomechanics, 2010. 43(1): p. 156-65.

22. Beck, M., et al., Hip morphology influences the pattern of damage to the acetabular cartilage: femoroacetabular impingement as a cause of early osteoarthritis of the hip. The Journal of bone and joint surgery. British volume, 2005. 87(7): p. 1012-8.

23. Ganz, R., et al., Femoroacetabular impingement: a cause for osteoarthritis of the hip. Clinical orthopaedics and related research, 2003(417): p. 112-20.

24. Keefe, M.W., et al., Radiofrequency cartilage reshaping: efficacy, biophysical measurements, and tissue viability. Archives of facial plastic surgery, 2003. 5(1): p. 46-52.

25. Ho, K.H., et al., Electromechanical reshaping of septal cartilage. The Laryngoscope, 2003. 113(11): p. 1916-21.

26. Williams, G.M., J.W. Lin, and R.L. Sah, Cartilage reshaping via in vitro mechanical loading. Tissue engineering, 2007. 13(12): p. 2903-11.

27. Balcom, N.T., et al. Matrix Remodeling Accompanies in vitro Articular Cartilage Shaping. in ASME SBC. 2013. Sunriver, Oregon: ASME.

28. Palmer, A.W., R.E. Guldberg, and M.E. Levenston, Analysis of cartilage matrix fixed charge density and three-dimensional morphology via contrast-enhanced microcomputed tomography. Proceedings of the National Academy of Sciences of the United States of America, 2006. 103(51): p. 19255-60.

29. Han, E.H., et al., Shaped, stratified, scaffold-free grafts for articular cartilage defects. Clinical orthopaedics and related research, 2008. 466(8): p. 1912-20.

30. Herbage, D., J. Bouillet, and J.C. Bernengo, Biochemical and physiochemical characterization of pepsin-solubilized type-II collagen from bovine articular cartilage. The Biochemical journal, 1977. 161(2): p. 303-12.

31. Pal, S., et al., Structural changes during development in bovine fetal epiphyseal cartilage. Collagen and related research, 1981. 1(2): p. 151-76.

32. Kim, Y.J., et al., Fluorometric assay of DNA in cartilage explants using Hoechst 33258. Analytical biochemistry, 1988. 174(1): p. 168-76.

33. Torzilli, P.A., Water content and equilibrium water partition in immature cartilage. Journal of orthopaedic research : official publication of the Orthopaedic Research Society, 1988. 6(5): p. 766-9.

34. Hardingham, T.E., The role of link-protein in the structure of cartilage proteoglycan aggregates. The Biochemical journal, 1979. 177(1): p. 237-47.

35. Williams, G.M., K.R. Gratz, and R.L. Sah, Asymmetrical strain distributions and neutral axis location of cartilage in flexure. Journal of biomechanics, 2009. 42(3): p. 325-30. 
36. Khan, I.M., et al., Cartilage integration: evaluation of the reasons for failure of integration during cartilage repair. A review. European cells \& materials, 2008. 16: p. 26-39.

37. Hwang, J., et al., In Vitro Calcification of Immature Bovine Articular Cartilage: Formation of a Functional Zone of Calcified Cartilage. Cartilage, 2010. 1(4): p. 287-297.

38. Gutierrez-Franco, J.D., et al., The Effects of Static Bending on Cell Distribution in Immature Bovine Articular Cartilage Shaped In Vitro, in BMES2012: Georgia.

39. Williams, G.M. and R.L. Sah, In vitro modulation of cartilage shape plasticity by biochemical regulation of matrix remodeling. Tissue engineering. Part A, 2011. 17(1-2): p. 17-23. 


\section{Appendix A: Project Data}

Table 11: Relaxed opening angle data.

\begin{tabular}{|c|c|c|c|c|c|c|c|c|}
\hline \multirow[b]{2}{*}{$\begin{array}{c}\text { Sample } \\
\text { Name }\end{array}$} & \multirow[b]{2}{*}{ Group } & \multirow[b]{2}{*}{$\begin{array}{c}\text { Initial } \\
\text { Opening } \\
\text { Angle }\left(^{\circ}\right)\end{array}$} & \multicolumn{6}{|c|}{ Relaxed Opening Angle $\left({ }^{\circ}\right)$} \\
\hline & & & Meas. 1 & Meas. 2 & Meas. 3 & Average & Stdev & $\begin{array}{l}\text { CV } \\
(\%) \\
\end{array}$ \\
\hline $\mathrm{k} 3 \mathrm{p} 5 \mathrm{c} 1$ & concave & 186.6 & 147 & 145 & 143 & 145 & 2.0 & 4.96 \\
\hline $\mathrm{k} 3 \mathrm{p} 7 \mathrm{c} 2$ & concave & 181.0 & 133 & 138 & 132 & 134 & 3.0 & 6.48 \\
\hline $\mathrm{k} 5 \mathrm{p} 9 \mathrm{c} 3$ & concave & 177.8 & 155 & 151 & 149 & 152 & 2.9 & 11.07 \\
\hline $\mathrm{k} 3 \mathrm{p} 3 \mathrm{x} 1$ & convex & 178.1 & 150 & 149 & 144 & 148 & 3.1 & 10.11 \\
\hline $\mathrm{k} 3 \mathrm{p} 8 \mathrm{x} 3$ & convex & 183.0 & 136 & 137 & 135 & 136 & 1.4 & 2.93 \\
\hline $\mathrm{k} 3 \mathrm{p} 9 \mathrm{c} 3$ & convex & 184.4 & 136 & 136 & 133 & 135 & 1.4 & 2.89 \\
\hline $\mathrm{k} 4 \mathrm{p} 10 \times 3$ & convex & 180.3 & 166 & 156 & 150 & 157 & 7.7 & 33.40 \\
\hline $\mathrm{k} 5 \mathrm{p} 2 \mathrm{x} 1$ & convex & 175.0 & 147 & 151 & 153 & 150 & 3.1 & 12.79 \\
\hline $\mathrm{k} 5 \mathrm{p} 6 \mathrm{x} 2$ & convex & 179.9 & 172 & 165 & 167 & 168 & 3.5 & 29.52 \\
\hline k6p1x1 & convex & 176.8 & 170 & 190 & 167 & 176 & 12.3 & 1028.2 \\
\hline k1p1z1 & D0 & 167.4 & 155 & 169 & 178 & 167 & 11.6 & N/A \\
\hline $\mathrm{k} 1 \mathrm{p} 2 \mathrm{z} 2$ & D0 & 178.9 & 178 & 179 & 180 & 179 & 0.7 & N/A \\
\hline $\mathrm{k} 2 \mathrm{p} 4 \mathrm{z} 1$ & D0 & 174.5 & 175 & 176 & 172 & 174 & 2.2 & N/A \\
\hline $\mathrm{k} 3 \mathrm{p} 2 \mathrm{z} 1$ & D0 & 180.8 & 185 & 169 & 189 & 181 & 10.7 & N/A \\
\hline $\mathrm{k} 4 \mathrm{p} 4 \mathrm{z} 1$ & D0 & 181.7 & 184 & 181 & 180 & 182 & 1.9 & N/A \\
\hline $\mathrm{k} 4 \mathrm{p} 9 \mathrm{z} 2$ & D0 & 172.0 & 174 & 174 & 167 & 172 & 4.0 & N/A \\
\hline k5p5z1 & D0 & 173.1 & 177 & 174 & 169 & 173 & 4.2 & N/A \\
\hline $\mathrm{k} 5 \mathrm{p} 7 \mathrm{z} 2$ & D0 & 182.3 & 192 & 174 & 181 & 182 & 9.0 & N/A \\
\hline k6p3z1 & D0 & 174.7 & 185 & 172 & 168 & 175 & 9.0 & N/A \\
\hline $\mathrm{k} 3 \mathrm{p} 1 \mathrm{f} 1$ & FS & 171.3 & 167 & 167 & 171 & 168 & 2.3 & N/A \\
\hline k3p6f2 & FS & 181.7 & 182 & 188 & 191 & 187 & 4.5 & N/A \\
\hline $\mathrm{k} 4 \mathrm{p} 1 \mathrm{f} 1$ & FS & 181.6 & 181 & 198 & 189 & 189 & 8.3 & N/A \\
\hline k4p6f2 & FS & 170.4 & 175 & 177 & 168 & 173 & 4.6 & N/A \\
\hline k5p10f3 & FS & 179.6 & 174 & 174 & 172 & 173 & 0.8 & N/A \\
\hline $\mathrm{k} 5 \mathrm{p} 3 \mathrm{f} 1$ & FS & 179.4 & 179 & 170 & 177 & 175 & 4.4 & N/A \\
\hline $\mathrm{k} 5 \mathrm{p} 8 \mathrm{f} 2$ & FS & 178.8 & 178 & 183 & 163 & 174 & 10.1 & N/A \\
\hline
\end{tabular}


Table 12: Biochemical data normalized to either initial wet weight (WWi) or final wet weight (WWf)

\begin{tabular}{|c|c|c|c|c|c|c|c|}
\hline $\begin{array}{c}\text { Sample } \\
\text { Name }\end{array}$ & Group & $\begin{array}{c}\text { PG } \\
(\% \\
\text { WWf }) \\
\end{array}$ & $\begin{array}{c}\text { PG } \\
(\% \\
\text { WWi }) \\
\end{array}$ & $\begin{array}{c}\text { COL } \\
(\% \\
\text { WWf })\end{array}$ & $\begin{array}{c}\text { COL } \\
(\% \\
\text { WWi })\end{array}$ & $\begin{array}{c}\text { Approximate } \\
\text { Cell Density } \\
\text { per WWf } \\
\left(10^{\wedge} 6 / g\right)\end{array}$ & $\begin{array}{c}\text { Approximate } \\
\text { Cell Density } \\
\text { per WWi } \\
\left(10^{\wedge} 6 / g\right)\end{array}$ \\
\hline $\mathrm{k} 3 \mathrm{p} 5 \mathrm{c} 1$ & concave & 4.45 & 4.96 & 9.41 & 10.48 & 46.61 & 51.93 \\
\hline $\mathrm{k} 3 \mathrm{p} 7 \mathrm{c} 2$ & concave & 4.39 & 4.80 & 9.18 & 10.02 & 113.43 & 123.85 \\
\hline $\mathrm{k} 5 \mathrm{p} 9 \mathrm{c} 3$ & concave & 3.09 & 3.07 & 6.96 & 6.90 & 33.10 & 32.85 \\
\hline $\mathrm{k} 3 \mathrm{p} 8 \mathrm{x} 3$ & convex & 3.37 & 3.70 & 7.37 & 8.07 & 76.09 & 83.36 \\
\hline k3p9c3 & convex & 4.01 & 4.56 & 5.75 & 6.53 & 82.67 & 93.90 \\
\hline $\mathrm{k} 4 \mathrm{p} 10 \mathrm{x} 3$ & convex & 4.46 & 4.77 & 10.69 & 11.44 & 51.35 & 54.92 \\
\hline $\mathrm{k} 5 \mathrm{p} 2 \mathrm{x} 1$ & convex & 4.45 & 4.66 & 6.32 & 6.62 & 73.31 & 76.82 \\
\hline k5p6x2 & convex & 4.64 & 4.67 & 8.62 & 8.68 & 58.74 & 59.10 \\
\hline k6p1x1 & convex & 3.11 & 3.08 & 9.80 & 9.70 & 91.69 & 90.81 \\
\hline k1p1z1 & D0 & 3.92 & 3.92 & 9.74 & 9.74 & 37.94 & 37.94 \\
\hline $\mathrm{k} 1 \mathrm{p} 2 \mathrm{z} 2$ & D0 & 4.87 & 4.87 & 10.29 & 10.29 & 93.51 & 93.51 \\
\hline $\mathrm{k} 2 \mathrm{p} 4 \mathrm{z} 1$ & D0 & 4.48 & 4.48 & 6.99 & 6.99 & 89.37 & 89.37 \\
\hline $\mathrm{k} 4 \mathrm{p} 4 \mathrm{z} 1$ & D0 & 3.24 & 3.24 & 5.53 & 5.53 & 20.92 & 20.92 \\
\hline k4p9z2 & D0 & 4.74 & 4.74 & 9.39 & 9.39 & 72.25 & 72.25 \\
\hline $\mathrm{k} 5 \mathrm{p} 5 \mathrm{z} 1$ & D0 & 3.82 & 3.82 & 11.18 & 11.18 & 62.98 & 62.98 \\
\hline $\mathrm{k} 5 \mathrm{p} 7 \mathrm{z} 2$ & D0 & 4.89 & 4.89 & 5.88 & 5.88 & 102.55 & 102.55 \\
\hline k6p3z1 & D0 & 4.69 & 4.69 & 10.75 & 10.75 & 80.10 & 80.10 \\
\hline k3p1f1 & FS & 3.53 & 4.54 & 8.61 & 11.09 & 85.93 & 110.59 \\
\hline k3p6f2 & FS & 5.11 & 6.01 & 7.02 & 8.25 & 8.94 & 10.52 \\
\hline k4p1f1 & FS & 3.88 & 4.48 & 7.40 & 8.54 & 71.14 & 82.12 \\
\hline k4p6f2 & FS & 3.40 & 3.98 & 5.13 & 5.99 & 88.49 & 103.42 \\
\hline $\mathrm{k} 5 \mathrm{p} 3 \mathrm{f} 1$ & FS & 3.08 & 3.37 & 10.11 & 11.08 & 68.92 & 75.52 \\
\hline $\mathrm{k} 5 \mathrm{p} 8 \mathrm{f} 2$ & FS & 4.50 & 5.09 & 7.91 & 8.94 & 84.76 & 95.75 \\
\hline
\end{tabular}


Table 13: Hexabrix data for superficial (S), middle (M), and deep (D) layers. *Samples labeled by "Sample \#\#" indicate unknown sample name but known sample group due to a recording error.

\begin{tabular}{|l|l|c|c|c|}
\cline { 3 - 5 } \multicolumn{2}{c|}{} & \multicolumn{3}{c|}{ Hexabrix (\%) } \\
\hline Sample & Group & S & M & D \\
\hline \hline k3p5c1 & concave & 7.352 & 7.497 & 7.178 \\
\hline Sample15 & concave & 7.446 & 6.886 & 6.218 \\
\hline k3p3x1 & convex & 8.560 & 7.637 & 6.085 \\
\hline k3p9c3 & convex & 7.912 & 7.412 & 5.889 \\
\hline Sample01 & convex & 6.244 & 5.708 & 4.538 \\
\hline Sample02 & convex & 6.625 & 5.778 & 4.897 \\
\hline Sample04 & convex & 6.981 & 6.196 & 4.815 \\
\hline k1p1z1 & D0 & 7.597 & 7.460 & 6.258 \\
\hline k1p2z2 & D0 & 7.858 & 7.391 & 7.029 \\
\hline k2p4z1 & D0 & 9.062 & 7.944 & 6.755 \\
\hline k3p2z1 & D0 & 7.487 & 7.740 & 6.977 \\
\hline k1p5f1 & FS & 7.363 & 7.188 & 7.084 \\
\hline k2p5f1 & FS & 6.829 & 6.215 & 6.056 \\
\hline k3p6f2 & FS & 6.409 & 6.060 & 5.108 \\
\hline
\end{tabular}


Table 14: Orientation ( $\alpha$ ) data for superficial $(S)$, middle (M), and deep (D) layers. Red indicates excluded data due to processing error.

\begin{tabular}{|l|l|c|c|c|c|}
\cline { 3 - 6 } \multicolumn{2}{c|}{} & \multicolumn{4}{c|}{ Orientation $(\boldsymbol{\alpha})$} \\
\hline Sample & Group & S & M & D & Total \\
\hline \hline k3p5c1 & concave & -0.218 & 3.482 & 1.052 & 1.439 \\
\hline k3p7c2 & concave & -3.867 & -15.943 & -10.595 & -10.135 \\
\hline k5p9c3 & concave & -0.521 & 2.352 & 1.894 & 1.241 \\
\hline k3p9c3 & convex & -1.475 & -0.566 & 15.992 & 4.650 \\
\hline k3p8x3 & convex & -4.680 & 0.095 & 16.380 & 3.932 \\
\hline k4p10x3 & convex & -4.292 & -6.977 & -2.373 & -4.547 \\
\hline k1p1z1 & D0 & -11.324 & -16.469 & -15.811 & -14.535 \\
\hline k1p2z2 & D0 & -4.484 & -4.864 & -6.163 & -5.170 \\
\hline k5p5z1 & D0 & & & & \\
\hline k5p3f1 & FS & 29.470 & 26.563 & -21.762 & 11.424 \\
\hline k5p8f2 & FS & 10.052 & 12.093 & 6.167 & 9.438 \\
\hline k5p10f3 & FS & -23.103 & 0.301 & 9.705 & -4.366 \\
\hline
\end{tabular}

Table 15: Orientation standard deviation ( $\alpha$ SD) for superficial (S), middle (M) and deep (D) layers. Red indicates excluded data due to processing error.

\begin{tabular}{|l|l|c|c|c|c|}
\cline { 3 - 6 } \multicolumn{2}{c}{} & \multicolumn{4}{c|}{$\alpha$ Standard Deviations } \\
\hline Sample & Group & S & M & D & Total \\
\hline \hline k3p5c1 & concave & 40.042 & 32.417 & 11.163 & 27.874 \\
\hline k3p7c2 & concave & 34.494 & 25.513 & 16.520 & 25.509 \\
\hline k5p9c3 & concave & 62.405 & 59.479 & 21.332 & 47.739 \\
\hline k3p9c3 & convex & 22.468 & 27.392 & 56.027 & 35.295 \\
\hline k3p8x3 & convex & 19.724 & 31.350 & 34.121 & 28.398 \\
\hline k4p10x3 & convex & 28.573 & 23.000 & 28.321 & 26.631 \\
\hline k1p1z1 & D0 & 13.421 & 9.338 & 7.072 & 9.944 \\
\hline k1p2z2 & D0 & 30.071 & 32.730 & 18.145 & 26.982 \\
\hline k5p5z1 & D0 & & & & \\
\hline k5p3f1 & FS & 45.810 & 33.295 & 10.000 & 29.702 \\
\hline k5p8f2 & FS & 47.035 & 52.574 & 52.286 & 50.631 \\
\hline k5p10f3 & FS & 15.483 & 26.001 & 31.724 & 24.402 \\
\hline
\end{tabular}


Table 16: Parallelism Index data for superficial (S), middle (M), and deep (D) layers

\begin{tabular}{|l|l|c|c|c|c|}
\cline { 3 - 6 } \multicolumn{2}{c|}{} & \multicolumn{4}{c|}{ Parallelism Index (PI) } \\
\hline Sample & Group & S & M & D & Total \\
\hline \hline k3p5c1 & concave & 0.571 & 0.624 & 0.655 & 0.617 \\
\hline k3p7c2 & concave & 0.592 & 0.656 & 0.670 & 0.639 \\
\hline k5p9c3 & concave & 0.395 & 0.404 & 0.539 & 0.446 \\
\hline k3p9c3 & convex & 0.499 & 0.573 & 0.525 & 0.532 \\
\hline k3p8x3 & convex & 0.569 & 0.494 & 0.477 & 0.513 \\
\hline k4p10x3 & convex & 0.365 & 0.475 & 0.468 & 0.436 \\
\hline k1p1z1 & D0 & 0.620 & 0.757 & 0.723 & 0.700 \\
\hline k1p2z2 & D0 & 0.514 & 0.515 & 0.600 & 0.543 \\
\hline k5p5z1 & D0 & 0.604 & 0.579 & 0.622 & 0.601 \\
\hline k5p3f1 & FS & 0.658 & 0.716 & 0.728 & 0.701 \\
\hline k5p8f2 & FS & 0.627 & 0.589 & 0.506 & 0.574 \\
\hline k5p10f3 & FS & 0.435 & 0.486 & 0.653 & 0.525 \\
\hline
\end{tabular}

Table 17: Parallelism Index Standard Deviation (PISD) data for superficial (S), middle (M) and deep (D) layers.

\begin{tabular}{|l|l|c|c|c|c|}
\cline { 3 - 6 } \multicolumn{2}{c|}{} & \multicolumn{4}{c|}{ PI Standard Deviations } \\
\hline Sample & Group & S & M & D & Total \\
\hline \hline k3p5c1 & concave & 0.109 & 0.122 & 0.130 & 0.120 \\
\hline k3p7c2 & concave & 0.139 & 0.094 & 0.110 & 0.114 \\
\hline k5p9c3 & concave & 0.198 & 0.178 & 0.251 & 0.209 \\
\hline k3p9c3 & convex & 0.113 & 0.102 & 0.089 & 0.101 \\
\hline k3p8x3 & convex & 0.136 & 0.099 & 0.104 & 0.113 \\
\hline k4p10x3 & convex & 0.190 & 0.125 & 0.108 & 0.141 \\
\hline k1p1z1 & D0 & 0.337 & 0.182 & 0.118 & 0.213 \\
\hline k1p2z2 & D0 & 0.267 & 0.173 & 0.123 & 0.187 \\
\hline k5p5z1 & D0 & 0.112 & 0.103 & 0.155 & 0.123 \\
\hline k5p3f1 & FS & 0.169 & 0.154 & 0.095 & 0.139 \\
\hline k5p8f2 & FS & 0.108 & 0.088 & 0.080 & 0.092 \\
\hline k5p10f3 & FS & 0.189 & 0.137 & 0.102 & 0.143 \\
\hline
\end{tabular}


Table 18: Area fraction (AF) data for superficial (S), middle (M) and deep (D) layers based on the PI color map

\begin{tabular}{|l|l|c|c|c|c|}
\cline { 3 - 6 } \multicolumn{1}{c|}{} & \multicolumn{4}{c|}{ Mean Area Fraction (AF) from PI } \\
\hline Sample & Group & S & M & D & Total \\
\hline \hline k3p5c1 & concave & 0.589 & 0.747 & 0.893 & 0.743 \\
\hline k3p7c2 & concave & 0.496 & 0.683 & 0.823 & 0.667 \\
\hline k5p9c3 & concave & 0.307 & 0.272 & 0.648 & 0.409 \\
\hline k3p9c3 & convex & 0.417 & 0.794 & 0.744 & 0.652 \\
\hline k3p8x3 & convex & 0.527 & 0.537 & 0.479 & 0.514 \\
\hline k4p10x3 & convex & 0.315 & 0.739 & 0.853 & 0.636 \\
\hline k1p1z1 & D0 & 0.408 & 0.490 & 0.706 & 0.535 \\
\hline k1p2z2 & D0 & 0.389 & 0.368 & 0.627 & 0.461 \\
\hline k5p5z1 & D0 & 0.543 & 0.653 & 0.807 & 0.668 \\
\hline k5p3f1 & FS & 0.454 & 0.648 & 0.874 & 0.659 \\
\hline k5p8f2 & FS & 0.602 & 0.516 & 0.541 & 0.553 \\
\hline k5p10f3 & FS & 0.309 & 0.523 & 0.954 & 0.595 \\
\hline
\end{tabular}

Table 19: Area fraction standard deviation (AFSD) data for superficial (S), middle (M) and deep (D) layers based on the PI color map.

\begin{tabular}{|l|l|c|c|c|c|}
\cline { 3 - 6 } \multicolumn{2}{c}{} & \multicolumn{4}{c|}{ AF Standard Deviations } \\
\hline Sample & Group & S & M & D & Total \\
\hline \hline k3p5c1 & concave & 0.257 & 0.243 & 0.163 & 0.221 \\
\hline k3p7c2 & concave & 0.306 & 0.244 & 0.207 & 0.252 \\
\hline k5p9c3 & concave & 0.291 & 0.210 & 0.356 & 0.286 \\
\hline k3p9c3 & convex & 0.243 & 0.199 & 0.182 & 0.208 \\
\hline k3p8x3 & convex & 0.276 & 0.261 & 0.245 & 0.261 \\
\hline k4p10x3 & convex & 0.280 & 0.273 & 0.180 & 0.244 \\
\hline k1p1z1 & D0 & 0.392 & 0.289 & 0.284 & 0.322 \\
\hline k1p2z2 & D0 & 0.374 & 0.273 & 0.281 & 0.309 \\
\hline k5p5z1 & D0 & 0.238 & 0.215 & 0.197 & 0.217 \\
\hline k5p3f1 & FS & 0.272 & 0.342 & 0.100 & 0.238 \\
\hline k5p8f2 & FS & 0.249 & 0.232 & 0.142 & 0.207 \\
\hline k5p10f3 & FS & 0.345 & 0.259 & 0.230 & 0.278 \\
\hline
\end{tabular}

